\title{
PALEO
}

Revue d'archéologie préhistorique

19 | 2007

Spécial table ronde (1 ère partie) : Le Gravettien : entités régionales d'une paléoculture européenne, Les Eyzies, juillet 2004

\section{Le Gravettien de Moldavie (30 000-23 000 BP)}

Gravettian in Moldavia.

\section{Pierre Noiret}

\section{OpenEdition}

\section{Journals}

Édition électronique

URL : https://journals.openedition.org/paleo/592

DOI : $10.4000 /$ paleo.592

ISSN : 2101-0420

Éditeur

SAMRA

Édition imprimée

Date de publication : 30 décembre 2007

Pagination : 159-179

ISSN : 1145-3370

Référence électronique

Pierre Noiret, « Le Gravettien de Moldavie (30 000-23 000 BP) », PALEO [En ligne], 19| 2007, mis en ligne le 23 avril 2009, consulté le 25 juin 2021. URL : http://journals.openedition.org/paleo/592 ; DOI https://doi.org/10.4000/paleo.592

\section{(c) (1)}

PALEO est mis à disposition selon les termes de la licence Creative Commons Attribution - Pas d'Utilisation Commerciale - Pas de Modification 4.0 International. 


\title{
LE GRAVETTIEN DE MOLDAVIE (30 000-23000 BP)
}

\author{
Pierre NOIRET ${ }^{(1)}$
}

\begin{abstract}
Résumé : En Moldavie, trois séquences de référence permettent de fixer le cadre chronologique dans lequel ont pris place les occupations gravettiennes (et épigravettiennes). Deux stades techniques ont été reconnus, montrant un développement autonome, très constant dans ses manifestations techniques et typologiques. II s'agit d'un Gravettien à grandes lames retouchées puis à pointes à cran (29 500 23000 BP), qui seront suivis d'un Épigravettien ancien (20 000 - 17000 BP), puis d'un Épigravettien récent (13 500-11 000 BP).

Mots-clés : Moldavie, Gravettien, Épigravettien, Molodovien.

Abstract: Gravettian in Moldavia. In Moldavia, three sequences serve as the basis for the chronological context for Gravettian (and Epigravettian) occupations. Two technological phases have been identified, showing independent development, homogenous in both technological and typological attributes. The Gravettian (20,000-17,000 BP) is characterized by large retouched blades, followed by shouldered points $(29,500-23,000 \mathrm{BP})$, succeeded by the Early Epigravettian $(20,000-17,000 \mathrm{BP})$ then by the Late Epigravettian $(13,500-11,000 \mathrm{BP})$.
\end{abstract}

Keywords: Moldavia, Gravettian, Epigravettian, Molodovian.

\section{1 - INTRODUCTION}

\section{1 - Cadre géographique}

Quelques sites seulement constituent une base documentaire fiable pour l'étude du Gravettien en Moldavie ; ils se trouvent sur les territoires de la Roumanie, de l'Ukraine et de la République moldave. Leur répartition est centrée autour des bassins moyens du Prut et du Dniestr, dans les deux cas à proximité d'affleurements riches en silex de très bonne qualité (Muraru 1990).

Cette zone est bordée par les Carpates orientales (et l'Ukraine trans-carpatique) à l'est, par la Plaine russe à l'ouest, par les Balkans, la mer Noire et les collines de Crimée au sud, et par le Plateau de Volhynie-Podolie au nord (fig. 1).

\section{2 - Le Gravettien en Europe}

Traditionnellement, on considère que le techno-complexe gravettien couvre la seconde partie du Pléniglaciaire moyen et le Pléniglaciaire supérieur, jusqu'au début de la récession de l'inlandsis. À travers toute l'Europe, il est caractérisé par un ensemble de traits technologiques communs : débitage laminaire à partir de nucléus à un ou deux plans de frappe opposés, production de lames minces et régulières, retouche abrupte appliquée aux pointes et aux ammatures. Après la transgression maximale de l'inlandsis, la tradition des outillages à dos abattu se poursuit en Europe centrale et orientale, sous la forme d'un Gravettien récent (ou Épigravettien), aux caractéristiques régionales plus développées et accentuées.

Récemment, une synthèse publiée par I.A. Borziac et V. Chirica (1999) a mentionné un nombre de 500 localités liées au Gravettien entre les Carpates et le Dniestr, dont quelquesunes sont décrites dans quelques synthèses (Chetraru 1973; Chernysh 1973 ; Chirica 1989 ; Borziac 1994). Ce Gravettien est dénommé localement “ Molodovien ".

\section{3 - Le Molodovien}

Le Gravettien à l'est des Carpates a d'abord suscité des travaux fondés sur l'exemple des études occidentales : ainsi, les chercheurs ukrainiens ont-ils élaboré des schémas d'évolution des industries du Paléolithique supérieur en six étapes, pour des ensembles essentiellement gravettiens (Boriskovsky 1953 ; Chernysh 1973), alors que les chercheurs roumains divisaient le Gravettien (comme l'Aurignacien) en une succession de phases évolutives en nombre variable selon les auteurs (Nicolaescu-Plopsor et al. 1966 ; Paunescu 1970).

Des tentatives de réduction du nombre de ces phases ont ensuite été proposées. Selon G.P. Grigor'ev (1970), deux phases évolutives de Paléolithique supérieur de 


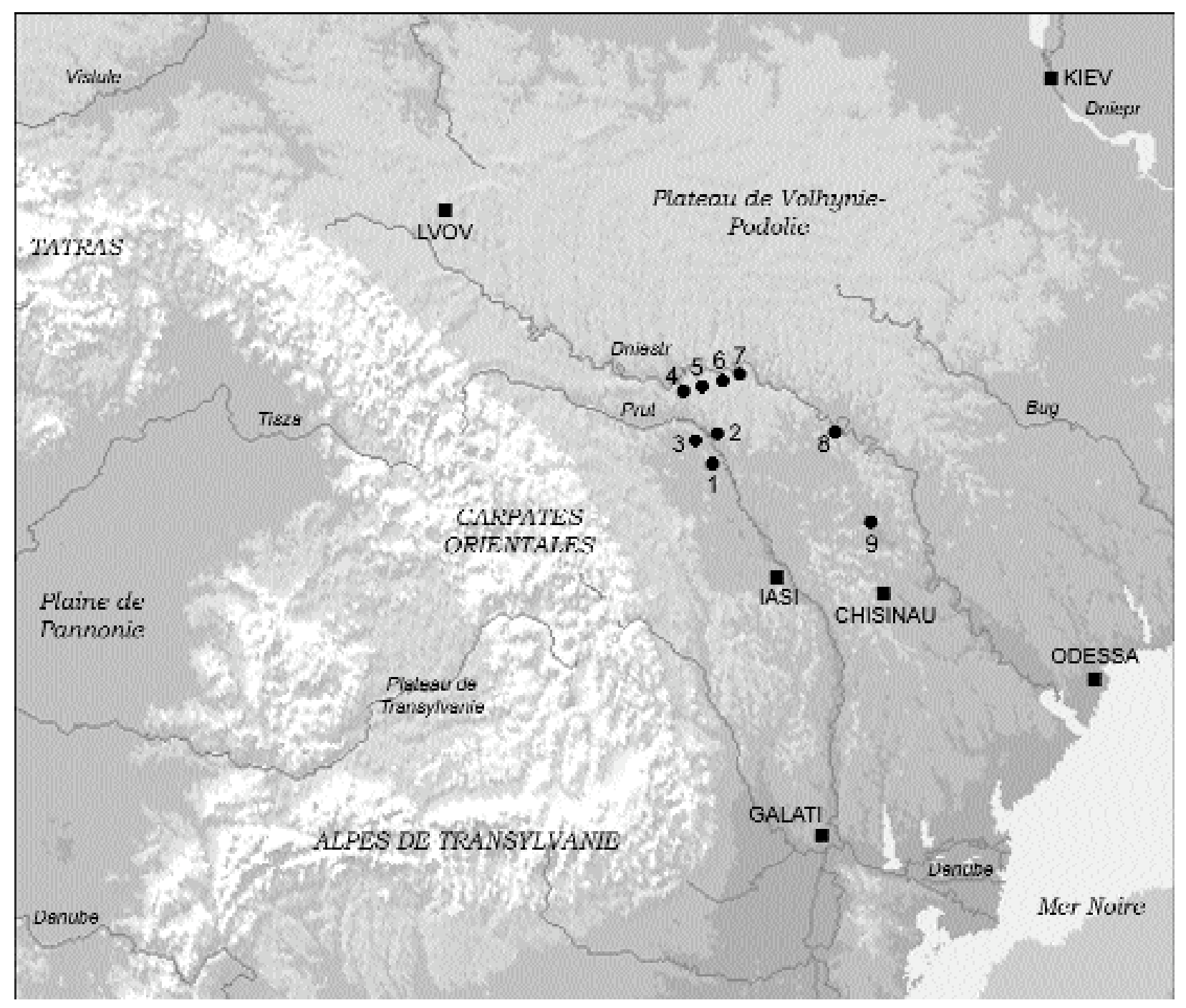

Figure 1 - Localisation des principaux sites gravettiens de Moldavie. Mitoc-Malu Galben (1), Ciuntu (2), Cotu-Miculinti (3), Molodova V (4), Korman IV (5), Babin I (6), Voronovitsa I (7), Cosauti (8), Ciutulesti I (9).

Figure 1- Location of the main Gravettian sites in Moldavia. Mitoc-Malu Galben (1), Ciuntu (2), Cotu-Miculinti (3), Molodova V (4), Korman IV (5), Babin I (6), Voronovitsa I (7), Cosauti (8), Ciutulesti I (9).

type Molodovien sont présentes dans la séquence classique de Molodova $\mathrm{V}$ : elles correspondent aux niveaux 10-7, puis aux niveaux 6-1.

De la même manière, Fl. Mogosanu $(1983,1986)$ distingue deux cycles dans le Gravettien de la Moldavie roumaine, lequel reste très probablement lié à celui du Dniestr moyen. Le premier cycle prend place durant les oscillations d'Ohaba B et Herculane I (en trois étapes), le second pendant l'oscillation d'Herculane II et au début de l'Holocène (en deux étapes). Ces étapes correspondraient à " autant de pénétrations ou de poussées saisonnières depuis le nord-est ", mais entre les deux cycles, un hiatus d'occupation est partout perceptible. Le second cycle est plus étendu dans l'espace et sans doute dans le temps. II est interprété comme la conséquence d'une " explosion démographique " et correspond à l'Épigravettien.
Un nombre restreint de datations radiométriques a pendant longtemps poussé les chercheurs roumains à placer le début du Gravettien vers 23000 BP (Mogosanu 1983, p. 45 ; Paunescu 1989, p. 140).

La séquence de référence est celle de Molodova $V$; elle se décompose en trois unités principales : les niveaux 10 à 8 , le niveau 7 (isolé en raison de la présence de pointes à cran) et les niveaux 6 à 1, postérieurs à $20000 \mathrm{BP}$ et décrits comme épigravettiens (Kozlowski 1986, p. 191). Selon A.N. Rogachev et M.V. Anikovich (1984, p. 198), cette séquence montre deux cultures différentes : une culture dite "Moldovskaya" (niveaux 10 à 7), puis une culture dite "Dniestrovskaya " (niveaux 6 à 1). Des traits similaires caractérisent ces deux cultures mais il est, selon ces auteurs, peu probable qu'il y ait eu développement de 
l'une vers l'autre ; au contraire, les deux cultures se seraient plutôt développées en parallèle.

En réalité, les deux " cultures " proposées par A.N. Rogachev et M.V. Anikovich correspondent au Gravettien, puis à l'Épigravettien ; elles sont incontestablement distinguées par leur position chronostratigraphique, en succession, avant et après le dernier maximum glaciaire.

Aucune structure d'occupation très élaborée n'a été découverte dans le Molodovien et il semble que beaucoup de sites aient fonctionné, soit en tant que camps de base, soit en tant que camps satellites, indication peut-être de migrations saisonnières (Kozlowski 1986, p. 179).

En 1990, M. Otte avait publié une description du Gravettien d'Europe centrale en trois Stades techniques successifs, à partir de la séquence de Willendorf II (Otte 1990). Nous avons participé à l'application de cette description vers l'Europe orientale, en une succession de Stades caractérisés par des amatures n'apparaissant pas partout au même moment, mais toujours dans le même ordre (Otte et al. 1996a) ${ }^{2}$. I.A. Borziac et V. Chirica (1999) ont complété cette description en l'adaptant au Molodovien.

Trois sites de référence ont livré de longues séquences stratigraphiques aujourd'hui clairement attribuées d'un point de vue culturel (Molodova V : Paléolithique moyen, Gravettien et Épigravettien ; Mitoc-Malu Galben : Aurignacien et Gravettien ; Cosauti : Épigravettien), qui servent de fondement à notre connaissance du Gravettien local.

\section{2 - CADRE CHRONOSTRATIGRAPHIQUE}

La séquence de Molodova $V$ avait été décrite dès les années 1960 par I.K. Ivanova $(1959,1971,1987)$; celles de Mitoc-Malu Galben et de Cosauti n'avaient pas été traitées de manière aussi précise. Au final, un cadre chronostratigraphique exceptionnel est disponible pour les occupations aurignaciennes, gravettiennes et épigravettiennes reconnues dans ces trois gisements (Haesaerts et al. 1998, 2003, 2004), que l'on peut résumer comme suit (fig. 2).

Figure 2 - Cadre chronostratigraphique général à l'est des Carpates (Au : Aurignacien ; Gr : Gravettien ; Ep : Épigravettien) (d'après Haesaerts et al. 2003).

Figure 2 - General chronostratigraphic background east of the Carpatians (Au: Aurignacian; Gr: Gravettian: Ep:

Epigravettian) (after Haesaerts et al. 2003).

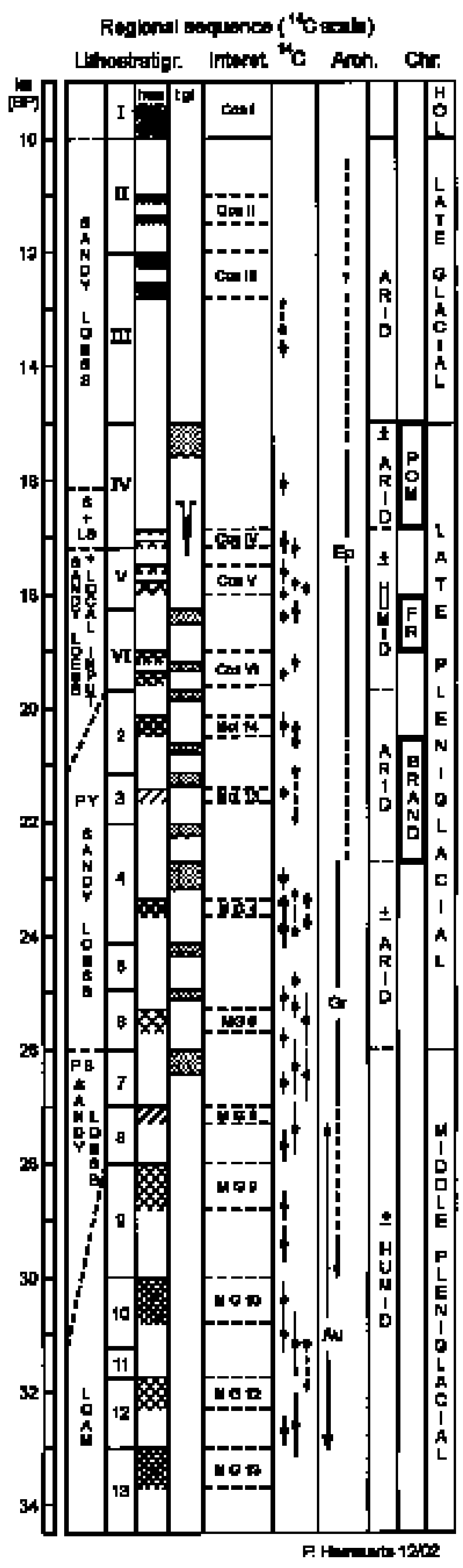

(2) Dans cette optique, le Stade I est caractérisé par la présence de microlithes, de fléchettes et de micro-gravettes (il n'existe pas à l'est des Carpates) ; le Stade II est caractérisé par des lames retouchées et appointées ; le Stade III est caractérisé par l'apparition des pointes à cran et le développement des pièces à retouche abrupte; le Stade IV ne correspond qu'à quelques ensembles dispersés entre 23000 et 20000 BP ; le Stade V correspond à des industries marquées par une microlithisation de l'outillage et par l'augmentation numérique des outils à retouche abrupte latérale. Ensuite, un Stade VI existe plus à l'est, à Mezin et à Mezhirich, mais pas en Moldavie; un ultime Stade VII correspond aux ensembles postérieurs à 14000 BP. 
La partie centrale de la séquence de Molodova $V$ appartient au Pléniglaciaire moyen ; elle est marquée par trois épisodes interstadiaires, vers 32650 BP, 30420 BP et 28 730 BP (les niveaux culturels gravettiens 10 et 9 se trouvant entre les deux derniers épisodes). I.K. Ivanova y distinguait deux composantes seulement, décrites comme le " sol du Dniestr". Ces épisodes sont suivis d'un rafraîchissement climatique traduit par un gley de toundra vers 26000 BP, marquant le début du Pléniglaciaire supérieur. La partie inférieure de la séquence du site (avec les niveaux culturels du Paléolithique moyen) reste problé matique, mais la partie supérieure montre les traces de trois horizons humifères liés à de brèves améliorations climatiques vers 25500 BP (avec le niveau culturel 8), vers $23700 \mathrm{BP}$ (la deuxième composante du niveau culturel 7 y est associée) et vers 20400 BP (avec le niveau culturel 6, épigravettien).

À Mitoc-Malu Galben, $14 \mathrm{~m}$ de sédiments correspondent à un enregistrement semi-continu entre 32730 et \pm 20000 BP. Entre 33000 et 26000 BP, les unités sédimentaires 13 à 7 correspondent à la seconde moitié du Pléniglaciaire moyen ; l'unité 6 , puis les unités 5 à 2 appartiennent à la première moitié du Pléniglaciaire moyen (entre \pm 26000 et $\pm 20000 \mathrm{BP})$.

Pour le Pléniglaciaire moyen, la signature du triple pédocomplexe de Molodova V est plus claire à Mitoc, où cinq épisodes positifs ont été enregistrés. Les deux premiers correspondent aux épisodes dénommés "MG 13 " (vers $33000 \mathrm{BP}$ ) et " MG 12 " (vers $31500 \mathrm{BP}$ ) ; ils sont équivalents au signal climatique de 32650 BP à Molodova V. La sédimentation éolienne démarre ensuite, vers $31000 \mathrm{BP}$; trois lœss sableux sont alors marqués de sols humifères d'intensité décroissante correspondant aux épisodes positifs dénommés " MG 10 " (vers 30000 BP), " MG 9 " (vers $28000 \mathrm{BP}$ ) et " MG 8 " (vers $27000 \mathrm{BP})$. Les principales occupations aurignaciennes de Mitoc ont été retrouvées juste avant et pendant l'épisode " MG 10 " (ensemble aurignacien I). Le Gravettien apparait plus tard, entre les deux derniers épisodes positifs (ensemble gravettien I ; voir ci-dessous). Un gley de toundra marque alors le début du Pléniglaciaire supérieur.

Le " sol de Briansk", souvent décrit dans la littérature d'Europe orientale, est isochrone au "sol du Dniestr ", tout en correspondant probablement à un processus de pédogenèse moins intense dans la Plaine russe que sur le Dniestr moyen (Gubin 1977 ; Hoffecker 1988). II s'agit d'un complexe pédologique " daté généralement entre 27 et 23 Kyr BP " (Kozlowski 2000), ce que confirment les datations radiométriques acceptées par A.V. Velichko pour ce sol dans la Plaine russe (entre 24900 et $22300 \mathrm{BP}$ ), où il est le marqueur de l'interstade de Briansk, entre 30000 et $25000 \mathrm{BP}$; il est fréquemment surmonté d'un horizon d'altération cryogénique (horizon de "Vladimir "), mis en place à la fin de la période, vers $23000-20000$ BP (Velichko et al. 1984). La forte pédogenèse enregistrée dans plusieurs séquences au début de cet interstade peut donc être de nature variée. Le sol de Briansk a été reconnu dans d'autres sites de Moldavie, mais pourrait correspondre à (au moins) deux événements distincts (vers 30.000 BP, correspondant à " MG 10 ", et vers 28000 BP, correspondant à “ MG 9 ") ; ce qui signifie que les ensembles culturels découverts en association avec ce sol ne sont certainement pas tous contemporains les uns des autres. Quoi qu'il en soit et pour revenir aux séquences de référence, le premier lœss sableux enregistre ensuite trois épisodes d'apports éoliens alternant avec trois gleys de toundra, à Mitoc comme à Molodova V. Dans cette succession, un léger horizon humifère daté de $25500 \mathrm{BP}$ à Molodova $V$ et une para-rendzine gris-brun datée de 23600 BP correspondent à deux épisodes positifs distincts, dénommés " MG 6 " et "MG 4 ", auxquels des occupations gravettiennes sont associées dans les deux sites (niveau 8 de Molodova $V$ et ensemble gravettien II de Mitoc, pour l'épisode " MG 6 " ; ensemble gravettien IV de Mitoc pour " MG 4 ").

Après une période de stabilisation du paysage sous permafrost, vers $23000 \mathrm{BP}$, la sédimentation lœssique s'est poursuivie jusque vers $20000 \mathrm{BP}$, dans l'environnement très sec d'un deuxième lœss sableux, où un court épisode climatique positif est marqué à Molodova $\mathrm{V}$ par un sol bioturbé, vers 21500 BP.

C'est à Cosauti que la phase finale du Pléistocène supérieur est la mieux documentée, entre \pm 20000 et 10000 BP, avec l'indication de conditions climatiques froides et plutôt sèches, puis entre 20000 et 17200 BP deux oscillations climatiques positives (" cos VI" et " cos V". Dans le Tardiglaciaire, apparaissent ensuite deux sols humifères, identifiables au complexe d'interstades de Bølling et Allerød, avant le dernier lœss sableux du Dryas III.

\section{3 - DATATIONS RADIOMÉTRIQUES}

Les datations radiométriques des ensembles gravettiens ont été obtenues essentiellement pour les sites de Molodova V et de Mitoc-Malu Galben (Haesaerts et al. $2003,2004)$, où elles sont intégrées à des séquences stratigraphiques bien décrites (tabl. 1).

À Molodova $\mathrm{V}, 12$ résultats radiométriques ont été obtenus récemment par $P$. Haesaerts, dans des unités sédimentaires non liées à des occupations humaines; ils ne seront pas directement pris en compte ici. II existe également 10 autres résultats anciens et nouveaux liés aux niveaux culturels 9,8 et 7 . Parmi ceux-ci, la date $n^{\circ} 8$ (niveau 8 ) peut être écartée (elle est infinie). II faut sans doute également écarter la date $n^{\circ} 9$ (la plus récente des deux existantes pour le niveau 9), car elle est en discordance stratigraphique avec d'autres résultats obtenus plus haut dans la séquence. Pour la même raison, la date $\mathrm{n}^{\circ} 1$ (niveau 7 ) est manifestement trop jeune et peut être abandonnée.

À Mitoc-Malu Galben, 43 datations radiométriques existent pour les ensembles gravettiens. Ces résultats, beaucoup plus nombreux, sont difficiles à appréhender sans triage. D'emblée, il est possible d'écarter les dates $n^{\circ} 17$, 19, 25 et 28 car elles ne sont pas finies et n'apportent rien à la connaissance de la chronologie des ensembles gravettiens. D'autres datations doivent être écartées, ainsi que l'ont expliqué Fr. Damblon et ses co-auteurs (Damblon et al. 1996, p. 193), pour diverses raisons. D'abord, de nombreuses dates GX correspondent à des échantillons de provenance douteuse et la prudence veut qu'elles soient écartées (dates $n^{\circ} 16,24,27,29,30,31,32$ et 42). D'autres datations GrN sont douteuses pour la même rai- 


\begin{tabular}{|c|c|c|c|c|}
\hline $\begin{array}{l}\mathbf{n}^{\circ} \\
1 \\
2 \\
3 \\
4 \\
5 \\
6 \\
7\end{array}$ & $\begin{array}{l}\text { Ensemble } \\
\text { Molodova V/7 } \\
\text { Molodova V/7 } \\
\text { Molodova V/7 } \\
\text { Molodova V/7 } \\
\text { Molodova V/7 } \\
\text { Molodova V/7 } \\
\text { Molodova V/7 } \\
\end{array}$ & $\begin{array}{c}\text { Date BP } \\
21.070 \pm 150 \\
23.000 \pm 170 \\
23.000 \pm 800 \\
23.700 \pm 320 \\
25.130+220 /-200 \\
25.170 \pm 120 \\
25.280 \pm 210\end{array}$ & $\begin{array}{l}\text { Matériau } \\
\text { os } \\
\text { charbon } \\
\text { charbon } \\
\text { sol } \\
\text { charbon } \\
\text { charbon } \\
\text { charbon }\end{array}$ & $\begin{array}{c}\text { Rèfèrence } \\
\text { GrA-9443 } \\
\text { GrA-9455 } \\
\text { MO-11 } \\
\text { GIN-10 } \\
\text { GrA-9564 } \\
\text { GrA-9457 } \\
\text { GrA-9456 }\end{array}$ \\
\hline 8 & Molodova V/8 & $>24.600$ & charbon & LU-14 \\
\hline 9 & Molodova V/9 & $28.100 \pm 1.000$ & charbon & LU-15B \\
\hline 10 & Molodova V/9 & $29.650 \pm 1.320$ & charbon & LU-15A \\
\hline 11 & Mitoc/Grav sup (2a) & $20.150 \pm 210$ & 05 & GrN-13765 \\
\hline 12 & Mitoc/Grav sup (2a) & $20.300 \pm 700$ & charbon & GrN-14031 \\
\hline 13 & Mitoc/Grav sup (3b) & $20.540 \pm 110$ & bois renne & GrA-5000 \\
\hline 14 & Mitoc/Grav IV (4a) & $23.850 \pm 100$ & charbon & GrA-1353 \\
\hline 15 & Mitoc/Grav IV (4b) & $23.650 \pm 400$ & 05 & OxA-1779 \\
\hline 16 & Mitoc/Grav IV (4b) & $24.620 \pm 810$ & charbon & $\mathrm{GX}-9422$ \\
\hline 17 & Mitoc /Grav IV (4b) & $>33.000$ & charbon & $\mathrm{GX}-8723$ \\
\hline 18 & Mitoc/Grav IV (5a) & $23.390 \pm 280$ & charbon & $\mathrm{GrN}-20438$ \\
\hline 19 & Mitoc/Grav IV (5a) & $>23.000$ & charbon & GrN-15448 \\
\hline 20 & Mitoc /Grav IV (5a) & $23.490 \pm 280$ & os & $\mathrm{GrN}-15 \mathrm{~B} 05$ \\
\hline 21 & Mitoc /Grav IV (5a) & $23.830 \pm 330$ & charbon & $\mathrm{GrN}-14034$ \\
\hline 22 & Mitoc /Grav IV (5a) & $24.650 \pm 450$ & os & OxA-1780 \\
\hline 23 & Mitoc /Grav IV (5a) & $27.150 \pm 750$ & charbon & $\mathrm{GrN}-12635$ \\
\hline 24 & Mitoc /Grav III (5b) & $19.910 \pm 990$ & charbon & GX-8724 \\
\hline 25 & Mitoc /Grav III (5b) & $=21.000$ & os & GX-9424 \\
\hline 26 & Mitoc /Grav III (5b) & $23.990 \pm 250$ & charbon & GrN-20439 \\
\hline 27 & Mitoc/Grav III (5b) & $24.820 \pm 850$ & charbon & GX-9425 \\
\hline 28 & Mitoc/Grav III (5b) & $\therefore 28.700$ & charbon & GX -8725 \\
\hline 29 & Mitoc /Grav III (6a) & $17.300+2100 /-1670$ & charbon & GX 9423 \\
\hline 30 & Mitoc /Grav III (6a) & $19.900+1050 /-930$ & os & GX 9429 \\
\hline 31 & Mitoc/Grav III (6a) & $20.945 \pm 850$ & charbon & GX 8503 \\
\hline 32 & Mitoc /Grav III (6a) & $22.050 \pm 1.250$ & charbon & $\mathrm{GX}-9420$ \\
\hline 33 & Mitoc /Grav II (6b) & $24.070 \pm 180$ & charbon & GrA-1020 \\
\hline 34 & Mitoc /Grav II (6b) & $25.140 \pm 210$ & charbon & $\mathrm{GrN}-14036$ \\
\hline 35 & Mitoc /Grav II (6b) & $25.610 \pm 220$ & charbon & $\mathrm{GrN}-15450$ \\
\hline 36 & Mitoc /Grav II (6b) & $25.610+500 /-470$ & charbon & $\mathrm{GrN}-20440$ \\
\hline 37 & Mitoc/Grav II (6b) & $26.100 \pm 800$ & charbon & GrN-15449 \\
\hline 38 & Mitoc /Grav II (6b) & $26.180 \pm 290$ & charbon & $\mathrm{GrN}-18811$ \\
\hline 39 & Mitoc /Grav II (6b) & $26.750 \pm 600$ & charbon & $\mathrm{GrN}-14035$ \\
\hline 40 & Mitoc/Grav II (6b) & $26.450 \pm 130$ & charbon & GrA-1354 \\
\hline 41 & Mitoc /Grav I (7a) & $25.840 \pm 90$ & 05 & $\mathrm{GrN}-15808$ \\
\hline 42 & Mitoc /Grav I (7a) & $26.700 \pm 1.040$ & charbon & GX-9418 \\
\hline 43 & Mitoc /Grav I (7b) & $23.070 \pm 180$ & 05 & GrN-13006 \\
\hline 44 & Mitoc /Grav I (7b) & $24.800 \pm 430$ & 05 & OxA-2033 \\
\hline 45 & Mitoc /Grav I (7b) & $25.330 \pm 420$ & charbon & $\mathrm{GrN}-14913$ \\
\hline 46 & Mitoc /Grav I (7b) & $26.500 \div 460 /-440$ & charbon & $\mathrm{GrN}-18 \mathrm{~B} 15$ \\
\hline 47 & Mitoc /Grav I (7b) & $26.020+650 /-600$ & charbon & $\mathrm{GrN}-18880$ \\
\hline 48 & Mitoc /Grav I (7b) & $26.380+800 /-500$ & charbon & GrN-18B81 \\
\hline 49 & Mitoc /Grav I (7b) & $26.300+450 /-430$ & charbon & GrN-18B79 \\
\hline 50 & Mitoc /Grav I (7b) & $25.080+500 /-470$ & charbon & $\mathrm{GrN}-18882$ \\
\hline 51 & Mitac /Grav I (7b) & $26.110+1050 /-930$ & charbon & $\mathrm{GrN}-18883$ \\
\hline 52 & Mitoc /Grav I (7b) & $27.500 \pm 600$ & os & OxA-1778 \\
\hline 53 & Mitoc /Grav I (7b) & $28.910 \pm 480$ & charbon & $\mathrm{GrN}-12636$ \\
\hline 54 & Korman IV/7 & $24.500 \pm 500$ & charbon & GIN-1099 \\
\hline 55 & Korman IV/7 & $25.140 \pm 350$ & charbon & LU-586 \\
\hline
\end{tabular}

Tableau 1 - Datations radiométriques pour le Gravettien de Moldavie.

Table 1 - Radiometric dates for the Gravettian of Moldavia. 
son (dates $n^{\circ} 20,34,35,37,39,41$ et 43 ). Enfin, il reste trois dates plus récentes que $23000 \mathrm{BP}$, pour des unités sédimentaires n'ayant pas livré d'ensembles assurément en place, ni diagnostiques et qui, pour ces raisons, peuvent être écartées ( $n^{\circ} 11,12$ et 13$)$.

Le site de Korman IV a également livré deux datations (Chemysh 1977 ; Ivanova 1977).

Deux constatations s'imposent. D'une part, tous les ensembles sont nettement postérieurs au niveau 9 de Molodova V ; d'autre part, le niveau 7 (à pointes à cran) de Molodova $V$ est bien constitué de deux composantes, vers 25000 et vers 23000 BP. Dans cette fourchette, viennent s'intercaler les ensembles gravettiens III et surtout IV de Mitoc (ce dernier avec un outillage lithique également marqué par les pointes à cran).

\section{4 - LE GRAVETTIEN ANCIEN DU PLÉNIGLACIAIRE MOYEN (35 000 - 27500 BP)}

Aucune donnée fiable ne signale la présence d'industries du Paléolithique supérieur (quelles qu'elles soient) avant 33000 BP en Moldavie. De même, il n'existe pas d'indication d'une persistance moustérienne au-delà de 40000 35000 BP. II semble que le territoire moldave ait connu une désaffection entre la fin du Paléolithique moyen et le début du Paléolithique supérieur. II s'agit peut-être d'une carence de la recherche et d'un manque de datations. Quoi qu'il en soit, dès $33000 \mathrm{BP}$, l'Aurignacien est attesté dans le bassin du Prut moyen, à Mitoc. Les premiers ensembles gravettiens apparaissent peu après (les niveaux 10 et 9 de Molodova V (sur le Dniestr moyen).

\section{1 - Position chronostratigraphique}

Les niveaux 10 et 9 de Molodova V relèvent du Gravettien ancien (Chernysh 1961, 1987 ; Otte 1981) et montrent que celui-ci apparaît entièrement constitué dans la phase froide immédiatement postérieure à l'interstade " MG 10 " (Haesaerts et al. 2003). Son origine n'est pas connue. La plupart des traits techno-typologiques particuliers à cette tradition culturelle sont présents : débitage laminaire à partir de nucléus volumétriques à un ou deux plans de frappe, produisant de grandes lames larges et régulières destinées à façonner les outils, dont de grandes lames appointées. La présence de petites armatures à dos abattu atteste une production lamellaire. Les outils caractéristiques sont les burins dièdres, les lames retouchées, les lames appointées, les micro-gravettes et les lamelles à dos simples (fig. 3).

Ces industries sont isolées et suivies d'un hiatus de deux mille ans, avant les premiers ensembles gravettiens (I et II) de Mitoc-Malu Galben, puis le niveau 8 de Molodova $\mathrm{V}$ (Otte et al. 1996a ; Haesaerts et al. 2003). Ce hiatus est actuellement difficile à comprendre, mais ne constitue pas un cas unique : il en existe un similaire à Willendorf II, en Europe centrale (Basse-Autriche), partiellement comblé par la présence de quelques silex épars entre les niveaux gravettiens 5 et 6 (Haesaerts et al. 1996), qui n'ont pas été rapportés par les fouilleurs à un " niveau culturel " particulier. De rares industries gravettiennes anciennes ont été découvertes, peut-être à Buran-Kaya III en Crimée
(Yanevich et al. 1996 ; Pettitt 1998), certainement à Temnata en Bulgarie (Kozlowski 1996), qui rendent ce Gravettien moldave ancien moins " isolé ", comme s'il était l'une des manifestations locales d'un phénomène global agissant à l'échelle du continent. Ces industries correspondent toutes à de petites installations proches dans le temps et dans l'espace d'installations aurignaciennes.

\section{3 - Origine}

La question de l'origine reste posée. Selon A.P. Chemysh (1959, 1973, 1985), il existerait une étape de transition locale menant du Paléolithique moyen vers ce Paléolithique supérieur, mais cette hypothèse de transition n'a jamais été argumentée et repose sur une industrie "transitionnelle" déplacée dans la séquence de Molodova $V$ (le niveau 10a). L'évolution aurait mené aux industries classiques du Gravettien de Molodova V, via les étapes " de Babin " et " de Voronovitsa", anciennes. Ces étapes sont peut-être anciennes, en effet, mais elles sont d'abord gravettiennes (Borziac et Chirica 1999 ; Noiret 2003-2004). Nous avons rappelé que G.P. Grigor'ev (1970) proposait de voir une succession en deux étapes dans le Molodovien, mais sans en argumenter la genèse. Quant à M.V. Anikovich (1992), il a vu l'origine de ce Molodovien dans le Szélétien, même s'il n'y a pratiquement pas de pointes szélétiennes dans le Molodovien.

Enfin, selon I.A. Borziac et L. Kulakovska (1998), le Gravettien ancien apparu tout constitué n'a pas son origine dans le Paléolithique moyen (les pièces " archaïques " y font défaut), ni dans une industrie transitionnelle de type Szélétien (pas de pointe foliacée) ou Bohunicien (pas de technologie Levallois) ; ils proposent plutôt d'en rechercher les racines dans l'Aurignacien de Mitoc ou de Bacho-Kiro (sans pièces bifaciales, ni technologie Levallois, donc similaire en cela au Gravettien), qui présenterait une "tendance à aménager les outils par retouche abrupte ", selon eux faible à Bacho-Kiro, plus nette à Mitoc. Ce Gravettien, dérivant donc d'un Aurignacien, aurait ensuite évolué selon le schéma proposé par M. Otte (Otte 1990 ; Otte et al. 1996a). Nous adhérons tout à fait à la fin de cette hypothèse, mais nous comprenons mal la référence à l'Aurignacien car aucune pièce aurignacienne de Mitoc ne montre une tendance à l'aménagement par retouche abrupte.

\section{5 - LE GRAVETTIEN DE LA FIN DU PLÉNIGLACIAIRE MOYEN ET DU DÉBUT DU PLÉNIGLACIAIRE SUPÉRIEUR (27 $500-23000 \mathrm{BP})$}

C'est le moment où les industries gravettiennes se développent, principalement le long du Dniestr moyen, sans doute en parallèle avec la plupart des industries à pièces bifaciales, réputées "transitionnelles " et concentrées autour du bassin du Prut moyen.

\section{1 - Localisation des ensembles}

Le Gravettien est attesté dans les bassins du Prut moyen et du Dniestr moyen, et dans le bassin du Raut. Sa répartition géographique et les vestiges retrouvés témoignent 

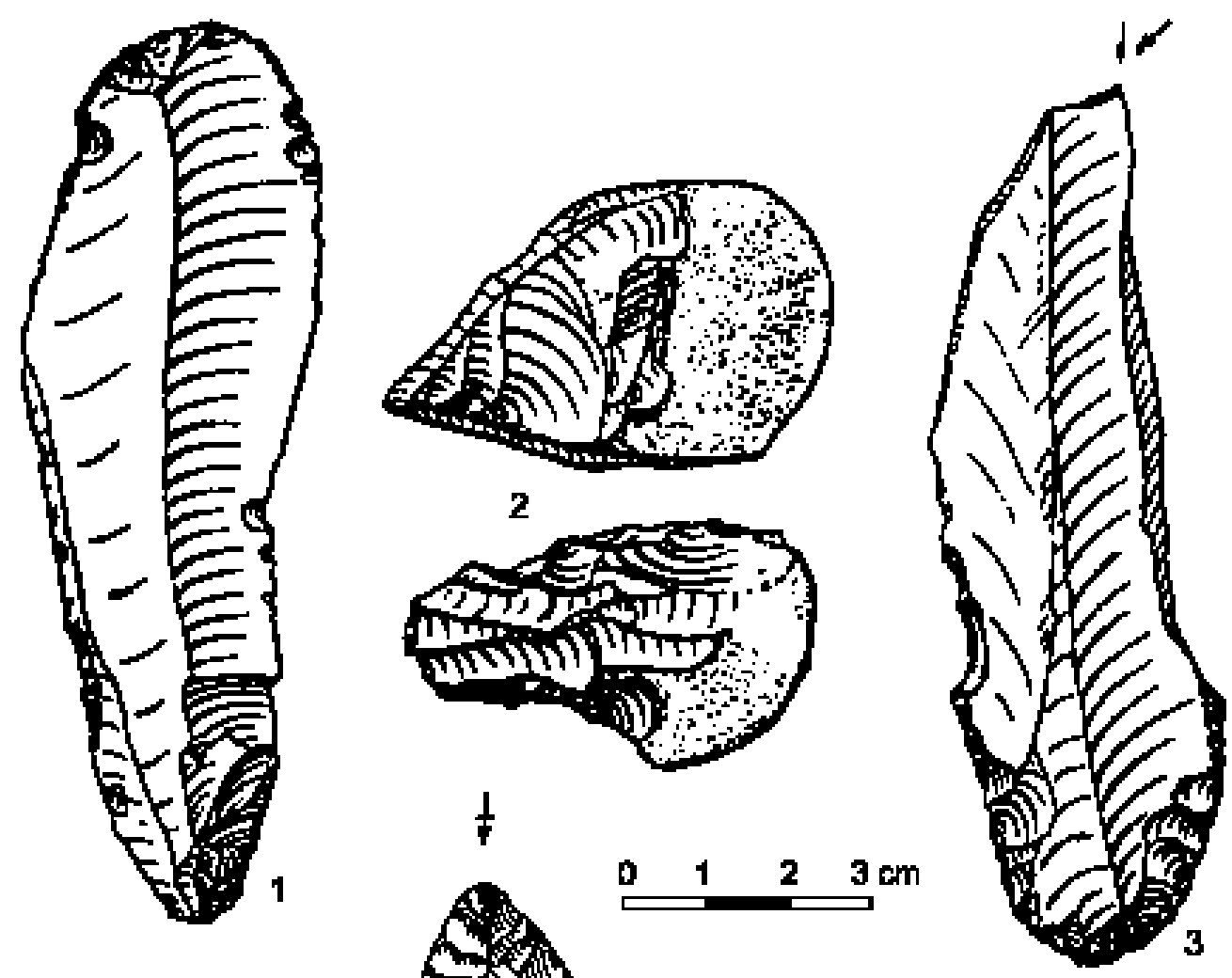

10

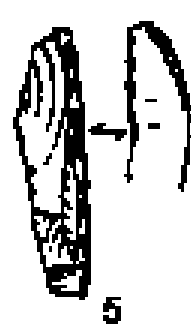

4

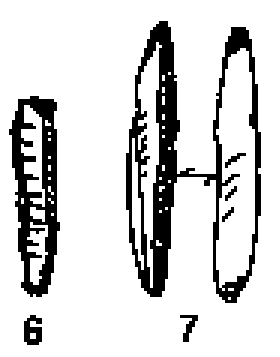

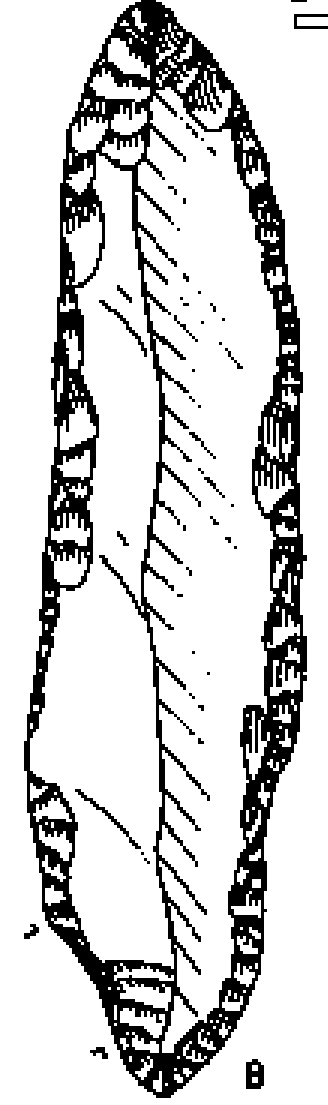
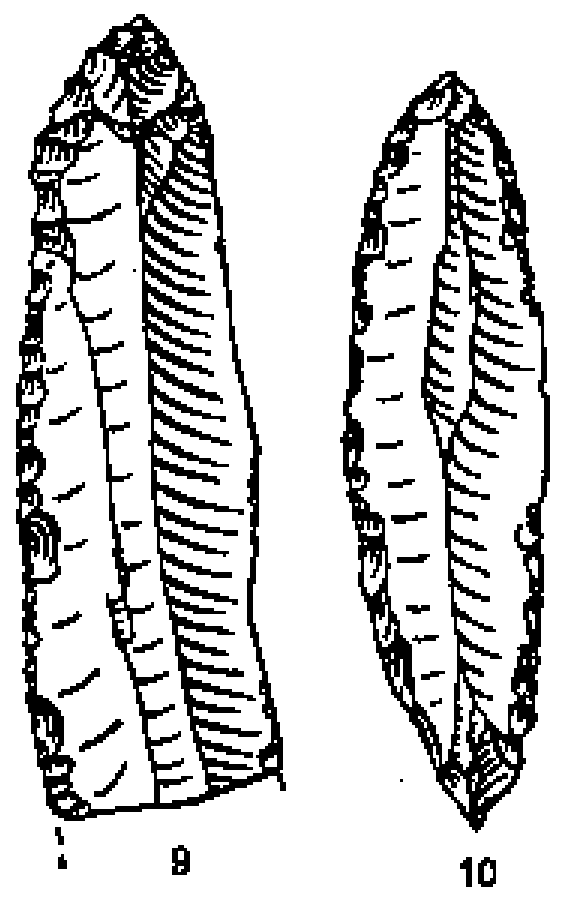

Figure 3 - Molodova V, niveau 10. Grattoir sur lame (1), grattoir caréné (2), grattoir-burin (3), lamelles à dos (4-6), micro-gravette (7), lames appointées (8-10) (d'après Chernysh 1961; Otte 1981).

Figure 3 - Molodova V, layer 10. End-scraper on blade (1), carinated end-scraper (2), end-scraper-burin (3), backed bladelets (4-6), micro-gravette (7), pointed blades (8-10) (after Chernysh 1961; Otte 1981). 
d'une stratégie développée d'occupation du territoire. Les principaux ensembles sont : les ensembles gravettiens I à IV de Mitoc-Malu Galben (Otte et al. 1996b, 2007) dans le bassin du Prut ; les niveaux 8-7 de Molodova V (Chemysh 1961, 1987 ; Otte 1981), les niveaux 7-6 de Korman IV (Chernysh 1977), les niveaux inférieur et médian de Babin I (Chemysh 1959), le niveau inférieur de Voronovitsa I (Chemysh 1959) dans le bassin du Dniestr ; et l'ensemble de Ciutulesti I (Borziac et Chetratu 1995) dans le bassin du Raut.

\section{2 - Position chronostratigraphique}

Les occupations débutent avec l'ensemble gravettien I de Mitoc-Malu Galben (immédiatement après 27000 BP) (fig. 4), puis se poursuivent entre 26500 et 25500 BP avec l'ensemble Il du même site, le niveau 8 de Molodova $V$ (fig. 5) et le niveau 7 de Korman IV, pendant l'oscillation climatique " MG 6 ", traduite à Mitoc et à Molodova $V$ par un petit sol humifère (Haesaerts et al. 2003), et à Korman IV par une malacofaune tempérée (Motuz 1977). Les niveaux inférieurs de Babin I (fig. 6) et de Voronovitsa I doivent sans doute être rapportés à l'une de ces deux phases, d'après leurs industries lithiques. Les conditions climatiques, globalement clémentes, correspondent à un environnement de steppe-forêt, cédant la place à une steppe froide à partir de $25000 \mathrm{BP}$ (après l'oscillation " MG 6 " et le début du Pléniglaciaire supérieur).

\section{3 - Restes fauniques}

Les restes fauniques comprennent principalement le Renne, le Cheval et le Mammouth (Chernysh 1959, 1987 ; Tatarinov 1977). Les données montrent une grande homogénéité le long du Dniestr. Même si certains ensembles correspondent probablement à des palimpsestes (à Babin I et à Voronovitsa I : enfouissement faible des vestiges, sur un terrain exposé, avec des indices d'érosion), tous ou presque indiquent l'existence d'une prédation orientée vers le cheval, puis vers le renne. Si ces deux espèces constituent la base de l'alimentation, d'autres grands herbivores y ont probablement participé, mais de manière plus ponctuelle, au premier rang desquels le bison. Les autres espèces, y compris des cervidés, ne sont chassés que de manière exceptionnelle ou ont fait l'objet de ramassages osseux (le rhinocéros). Le statut qu'il convient d'accorder au mammouth n'est pas clair ; il est rarement absent des ensembles gravettiens, mais sa représentation numérique est extrêmement variable (d'un ou de deux restes à plusieurs centaines, par exemple dans le niveau 7 de Molodova V). Des tests statistiques portant sur les restes fauniques (interprétation en termes de durées d'occupation) montrent que les occupations peuvent avoir été courtes, et le plus souvent de durée moyenne, fonctionnant sans doute par retours saisonniers de quelques semaines (Noiret 2003-2004).

Les deux sites localisés le long du Prut (Mitoc-Malu Galben) et du Raut (Ciutulesti I) sont différents. Dans les deux cas, la gamme des activités est beaucoup plus réduite : ce sont des sites d'ateliers. En conséquence, les activités de prédation y ont été secondaires. À Mitoc, le cheval domine, accompagné du bison ; le renne ne joue pas de rôle dans l'alimentation, sinon très secondaire (López Bayón et Gautier, 2007). À Ciutulesti I, le cheval est également accompagné du bison, mais aussi de quelques cervidés forestiers correspondant, soit à l'environnement immédiat du site, soit à la phase d'amélioration climatique au sein de laquelle la ou les occupations ont pris place (voire aux deux à la fois) (Borziac et Chetraru 1995).

\section{4 - Structures}

À côté des sites d'ateliers (Mitoc-Malu Galben, Ciutulesti I, en bord de rivière), des traces de petites huttes à foyer(s) furent découvertes dans les sites du Dniestr moyen, indiquant l'existence de petites installations résidentielles. On y trouve quelques broyeurs, pilons, outils en roches tenaces (peut-être destinés au broyage de végétaux) et une industrie osseuse parfois très riche (niveaux 8-7 de Molodova V, niveau 6 de Korman IV, niveau médian de Babin I).

\section{5 - Industrie lithique}

L'exploitation des ressources lithiques est locale. Des contacts existent avec les Carpates orientales (circulation de quelques lamelles en schiste noir d'Audia vers les bassins du Prut [ensemble gravettien I Mitoc ; Otte et al., 2007] et du Raut [Ciutulesti I ; Borziac et Chetraru 1995]), avec la Volhynie (circulation de rares lames en silex veiné de Volhynie vers les bassins du Prut [ensemble gravettien II de Mitoc] et du Dniestr [niveau 8 de Molodova V ; Kozlowski 1986]) et avec l'Europe centrale (circulation de pièces isolées en radiolarite vers le bassin du Dniestr [niveau inférieur de Babin I ; Chernysh 1959]). Ces quelques indications suggèrent une exploitation territoriale assez large.

Les nucléus et les modes de débitage présentent peu de variabilité. Les nucléus à lames sont prismatiques unipolaires, parfois bipolaires (à plans de frappe opposés, le plus souvent) et rarement à trois plans de frappe (ou plus). La régularité des nucléus - et donc des lames - varie d'un ensemble à l'autre, reflet de l'habileté des tailleurs et/ou de la morphologie des blocs, mais les dimensions des lames produites sont similaires (longueur moyenne comprise entre 7 et $15 \mathrm{~cm}$ ). Les blocs sont préparés sur les côtés et une crête est installée au centre de la surface d'éclatement, extraite en début d'exploitation. En l'absence de remontages, il est impossible de déterminer si l'orientation du débitage a pu changer au cours de l'exploitation, mais un souci de maintenance est constant (enlèvement de tablettes de réfection de plan de frappe, de flancs ou de lames "d'entretien" de section asymétrique). Une production annexe de lamelles était réalisée à partir de petits nucléus prismatiques ou de nucléus sur tranche d'éclat. En petit nombre, des nucléus à éclats accompagnaient parfois ces ensembles (circulaires d'exploitation centripète, ou discoïdes). Le grand pré-nucléus épais de forme ovale et à préparation bifaciale, caractéristique des ensembles gravettiens du niveau I de Kostenki 1, d'Avdeevo et de Zaraysk (Giria et Bradley 1998), n'apparaît pas en Moldavie, suggérant que les deux ensembles moldaves à pointes à cran (niveau 7 de Molodova V, ensemble gravettien IV de MitocMalu Galben) sont quelque peu différents de leurs contemporains d'Europe orientale. 

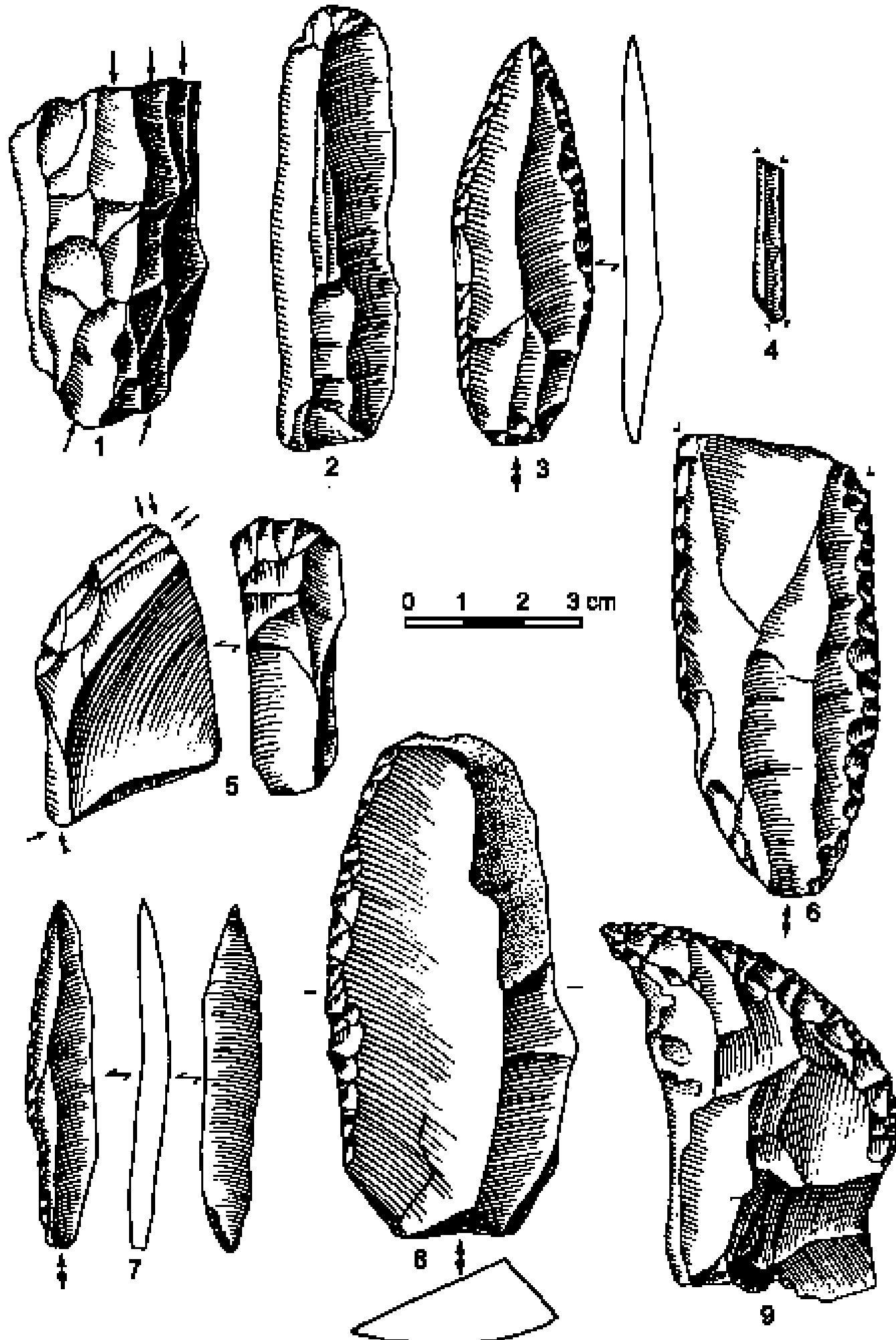

Figure 4 - Mitoc-Malu Galben, ensemble gravettien I. Nucléus à lames (1), grattoir sur lame (2), lame appointée (3), micro-gra vette en schiste noir d'Audia (4), burin mixte (5), base de lame retouchée (6), pointe à gibbosité (7), couteau (8), racloir déjeté (9) (d'après Otte et al. 2007).

Figure 4 - Mitoc-Malu Galben, Gravettian assemblage I. Blade core (1), end-scraper on blade (2), pointed blade (3), micro-gra vette on black schist from Audia (4), mixed burin (5), fragment of a retouched blade (6), pointe à gibbosité (7), knife (8), déjeté side-scraper (9) (after Otte et al. 2007). 


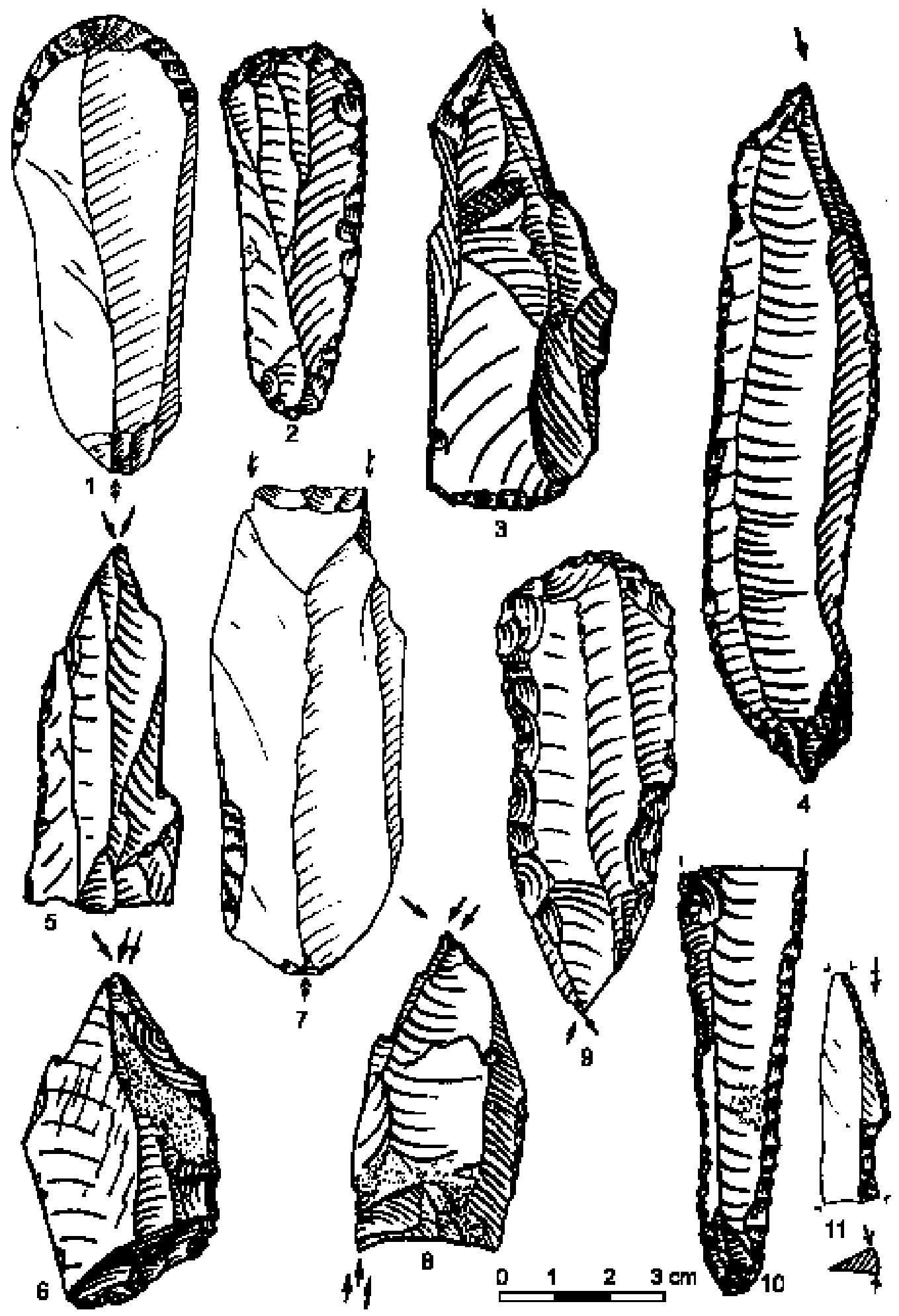

Figure 5 - Molodova V, niveau 8. Grattoir sur lame (1), grattoir sur lame retouchée (2), burin d'angle sur cassure (3), burins sur troncature retouchée (4, 7), burins dièdres (5-6), burin mixte (8), grattoir-burin (9), lame retouchée (10), pointe à cran (11) (d'après Chernysh 1961 ; Otte 1981).

Figure 5 - Molodova V, layer 8. End-scraper on blade (1), end-scraper on retouched blade (2), burin on break (3), burins on truncation (4, 7), dihedral burins (5-6), mixed burin (8), end-scraper-burin (9), retouched blade (10), shouldered point (11) (after Chernysh 1961; Otte 1981). 

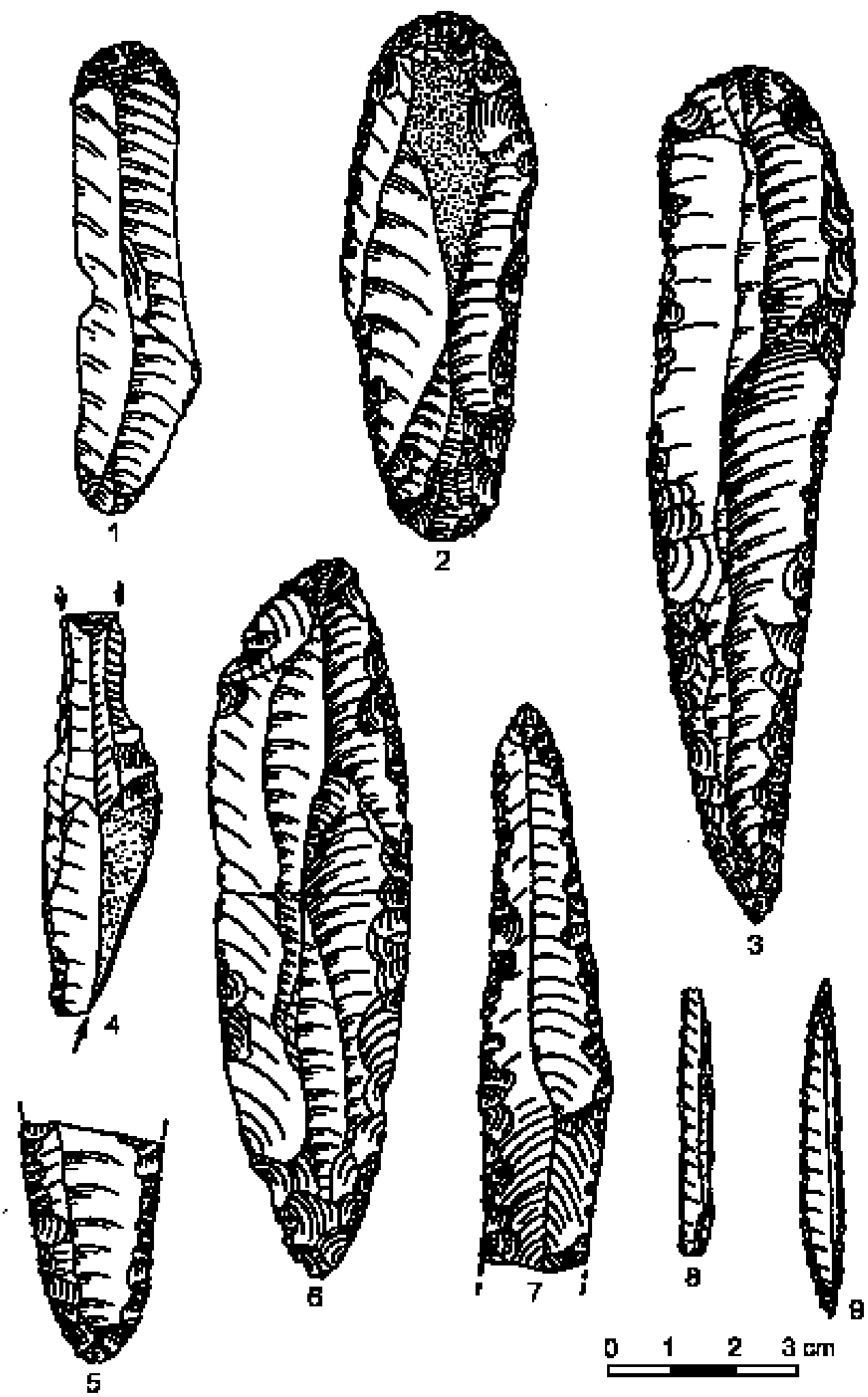

Figure 6 - Babin I, niveau inférieur. Grattoir sur lame (1), grattoir sur lame retouchée (2), grattoir sur lame appointée (3), burin sur cassure (4), base de lame retouchée (5), lames appointées (6-7), lamelle à dos (8), ; micro-gravette (9) (d'après Chernysh 1959).

Figure 6 - Babin I, lower layer. End-scraper on blade (1), end-scraper on retouched blade (2), end-scraper on pointed blade (3), burin on break (4), fragment of a retouched blade (5), pointed blades (6-7), backed bladelet (8), micro-gravette (9) (after Chernysh 1959). 

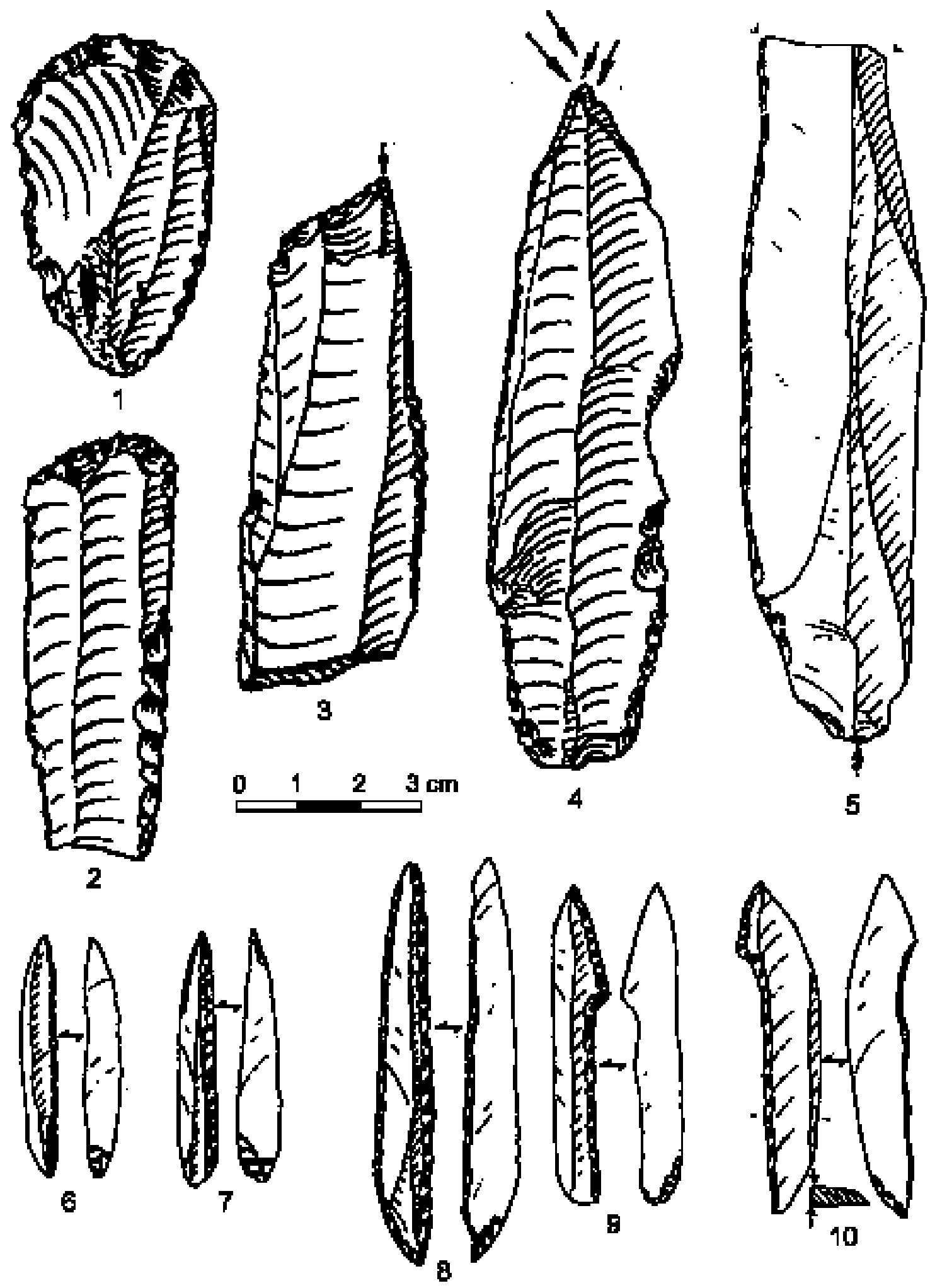

Figure 7 - Molodova V, niveau 7. Grattoir sur éclat (1), grattoir sur lame (2), burin sur troncature retouchée (3), burin dièdre (4), lame retouchée (5), micro-gravettes (6-7), pointe de La Gravette (8), pointes à cran (9-10) (d'après Chernysh 1961; Otte 1981).

Figure 7 - Molodova V, layer 7. End-scraper on flake (1), end-scraper on blade (2), burin on truncation (3), dihedral burin (4), retouched blade (5), micro-gravettes (6-7), La Gravette point (8), shouldered points (9-10) (after Chernysh 1961; Otte 1981). 

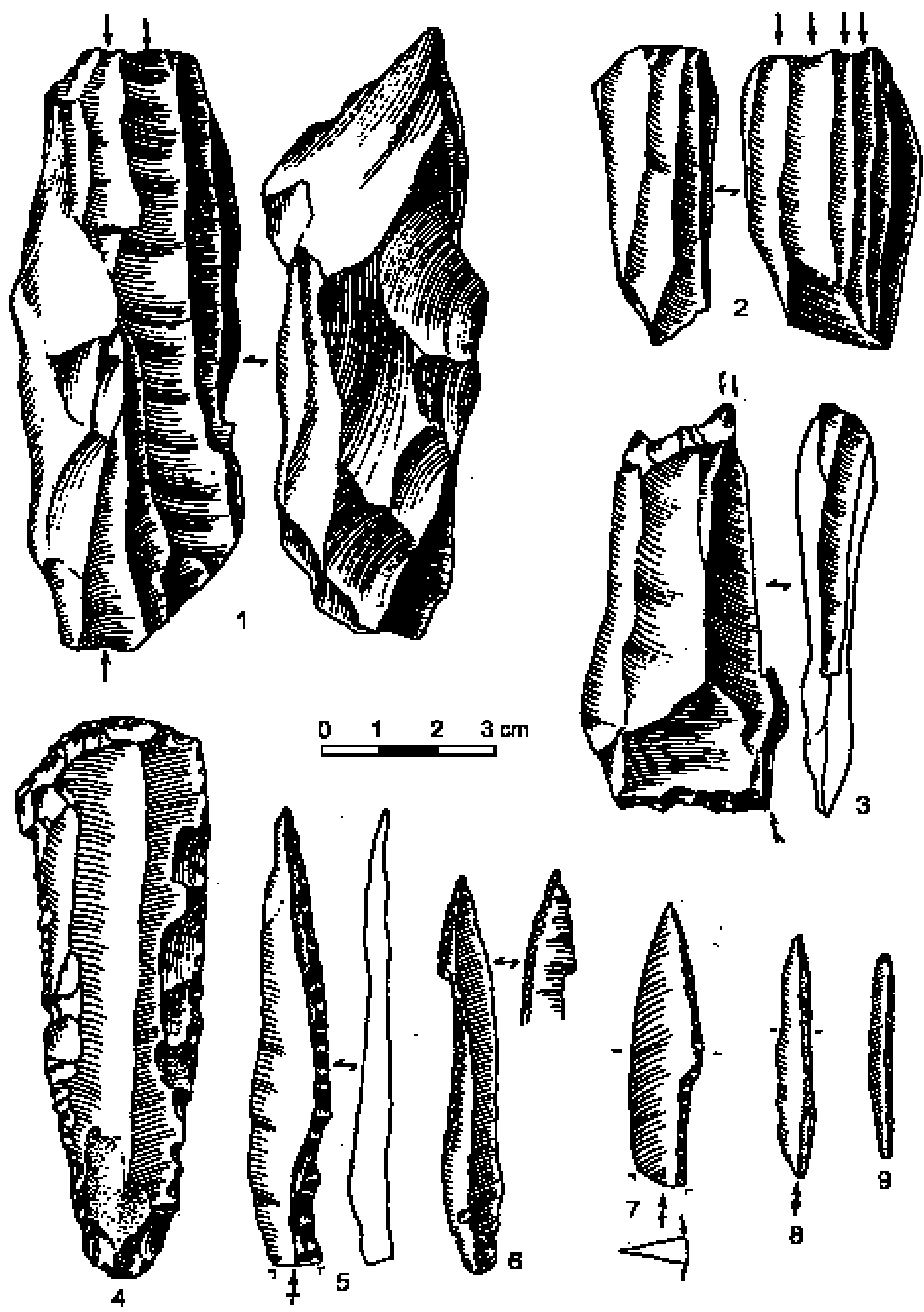
Les outils sont aménagés par retouche abrupte (pièces à dos), retouche semi-abrupte (lames retouchées, y compris des supports de grattoirs ou de burins) ou retouche plate (sur certaines lames retouchées ou appointées). La retouche inverse est rare et appliquée en tant qu'amincissement ventral. Les troncatures inverses sont exceptionnelles. Une très fine retouche plate ventrale est appliquée à quelques armatures, montrant des petits enlèvements inverses à une ou aux deux extrémités, peut-être destinés à corriger la rectitude du support (micro-gravettes, pointes à cran, pointe de La Gravette ou pointe à gibbosité).

Les industries contiennent surtout des burins, des lames retouchées et des grattoirs; les perçoirs et les couteaux de Kostenki sont toujours rares. Deux structures typologiques différentes existent, à burins, ou à lames retouchées. Ces deux outils sont toujours plus nombreux que les grattoirs et que les pièces à dos. Les lames appointées apparaissent en cinquième position. Le niveau 7 de Molodova $V$ diffère de ces deux schémas par un moins grand nombre de lames retouchées et une progression remarquable des armatures à dos (particulièrement nombreuses) ; les burins et les grattoirs sont cependant stables par rapport aux autres niveaux du site. Les pointes à cran constituent un repère chronologique précis : elles n'apparaissent pas avant 25.000 BP (et le niveau 7 de Molodova V) (fig. 7) et persistent jusque vers 23000 BP dans quelques ensembles : ensemble gravettien IV de Mitoc-Malu Galben (fig. 8), sans doute Zamostie I (Boriskovsky 1953 ; Kozlowski 1998).

Dans tous les cas, les grattoirs sur supports plats sont largement supérieurs en nombre aux éventuels grattoirs hauts aurignaciens (carénés, à museau, nucléiformes ; toujours rares). Les burins dièdres dominent toujours (sauf dans le niveau 6 de Korman IV), devant les burins sur cassure ou les burins sur troncature.

À Mitoc-Malu Galben, les grattoirs sont plus nombreux que les lames retouchées et que les burins dans les trois premiers ensembles. Dans le quatrième ensemble, les burins sont les plus nombreux, suivis des pièces à dos, des grattoirs et des lames retouchées (cette structure est similaire à celle du niveau 7 de Molodova $V$ ). Les burins dièdres dominent sur les burins sur cassure dans un premier temps (ensemble II), puis sur les burins sur troncature retouchée dans un second temps (ensemble IV). Ciutulesti I correspond au schéma dominé par les lames retouchées (puis les burins, puis les grattoirs) ; les burins sur troncature retouchée y sont par contre les plus nombreux.

L'analyse statistique (Analyse factorielle des correspondances sur les pourcentages des types d'outils) regroupe les industries à pointes à cran, mais distingue les industries antérieures en fonction de leur répartition géographique : bassin du Prut (grattoirs, lames appointées) et bassin du Dniestr (burins, lames retouchées, outils composites et perçoirs) (Noiret 2003-2004).

En fait, après le Gravettien ancien des niveaux 10-9 de Molodova $\mathrm{V}$, deux phases peuvent être distinguées, correspondant aux Stades II et III de M. Otte. De 27500 à 25000 $\mathrm{BP}$, existent des industries à burins (dièdres) plus nombreux que les grattoirs, à lames retouchées, appointées et tronquées, à lamelles à dos simples, à micro-gravettes et à armatures à dos abattu de grandes dimensions (pointes de La Gravette, pointes à gibbosité ; ensembles gravettiens I-III de Mitoc-Malu Galben, niveau 8 de Molodova V et probablement niveaux inférieur et médian de Babin I et niveau inférieur de Voronovitsa I). Ensuite, entre 25000 et 23000 $\mathrm{BP}$, apparaissent des industries où se développent les burins sur troncature retouchée et où apparaissent les pointes à cran (niveau 7 de Molodova $\mathrm{V}$, ensemble gravettien IV de Mitoc-Malu Galben), mais peut-être pas dans tous les sites (niveau 6 de Korman IV ; la possibilité d'ensembles contemporains de l'“ horizon à pointes à cran " sans pointes à cran renforce l'hypothèse selon laquelle la vague de peuplement à l'origine du Kostenkien n'a eu qu'un impact limité sur le Molodovien).

\section{6 - Industrie osseuse et manifestations esthétiques}

Les outils en matières organiques incluent des armatures autant que des objets domestiques façonnés (bâtons percés, manches en bois de renne, lissoirs) ou simplement aménagés de manière opportuniste (poinçons, " manches " sur ossement fendu, polissoirs). Les " pics " et " pioches " constituent un autre groupe, dont la fonction est mal connue, peut-être liée à l'exploitation de ressources végétales.

Les expressions artistiques communes au Pavlovien et au Wllendorfien-Kostenkien n'apparaissent pas en Moldavie : il n'existe aucune "Vénus ", ni statuette zoomorphe, mais simplement des pièces portant des incisions peu organisées, des représentations anthropomorphes schématiques et une amulette encochée (cette demière à Mitoc-Malu Galben [Chirica 1982], rappelant une pièce similaire découverte en Hongrie dans un contexte culturel identique, à Bodrogkerestúr-Henye [Dobosi 2000]).

\section{7 - Variabilité des occupations}

Le Gravettien moldave est remarquablement homogène entre 27500 et 23000 BP et ne semble pas avoir subi d'importantes influences pavloviennes ou kostenkiennes. Par rapport à la phase antérieure, il correspond à une nette prise de possession des territoires situés en bordure du Dniestr moyen. Les gisements étaient peut-être occupés à l'automne (lors du passage des troupeaux de rennes) et en été (pour la prédation d'autres grands herbivores) (Borziac et Chirica 1999). II n'existe pas de "super-sites " ou de sites d'agrégation, comme les grandes installations du Pavlovien, mais la pérennité des occupations est indiquée par des retours successifs aux mêmes emplacements.

I.A. Borziac (au contraire de A.P. Chernysh) insiste sur les différences sensibles entre ce Gravettien de Moldavie et le Pavlovien ou le Kostenkien, c'est-à-dire sur l'absence ou la très faible représentation en Moldavie d'outils tels que les couteaux de Kostenki, les pointes foliacées (ou les pointes à face plane), les micro-gravettes et, plus tard, les pointes de Kostenki, caractéristiques du Pavlovien puis du Kostenkien. Selon cet auteur (Borziac 1998 ; Borziac et Kulakovska 1998), ces différences seraient significatives d'une culture archéologique différente, propre au territoire compris entre les Carpates et le Bug (en fait, il s'agit du Molodovien). L'auteur ajoute que cette culture, peut-être mal affirmée avant 23000 BP (" macro-Gravettien "), est très clairement perceptible à partir de $20000 \mathrm{BP}$ (" microGravettien "), c'est-à-dire avec l'Épigravettien. 

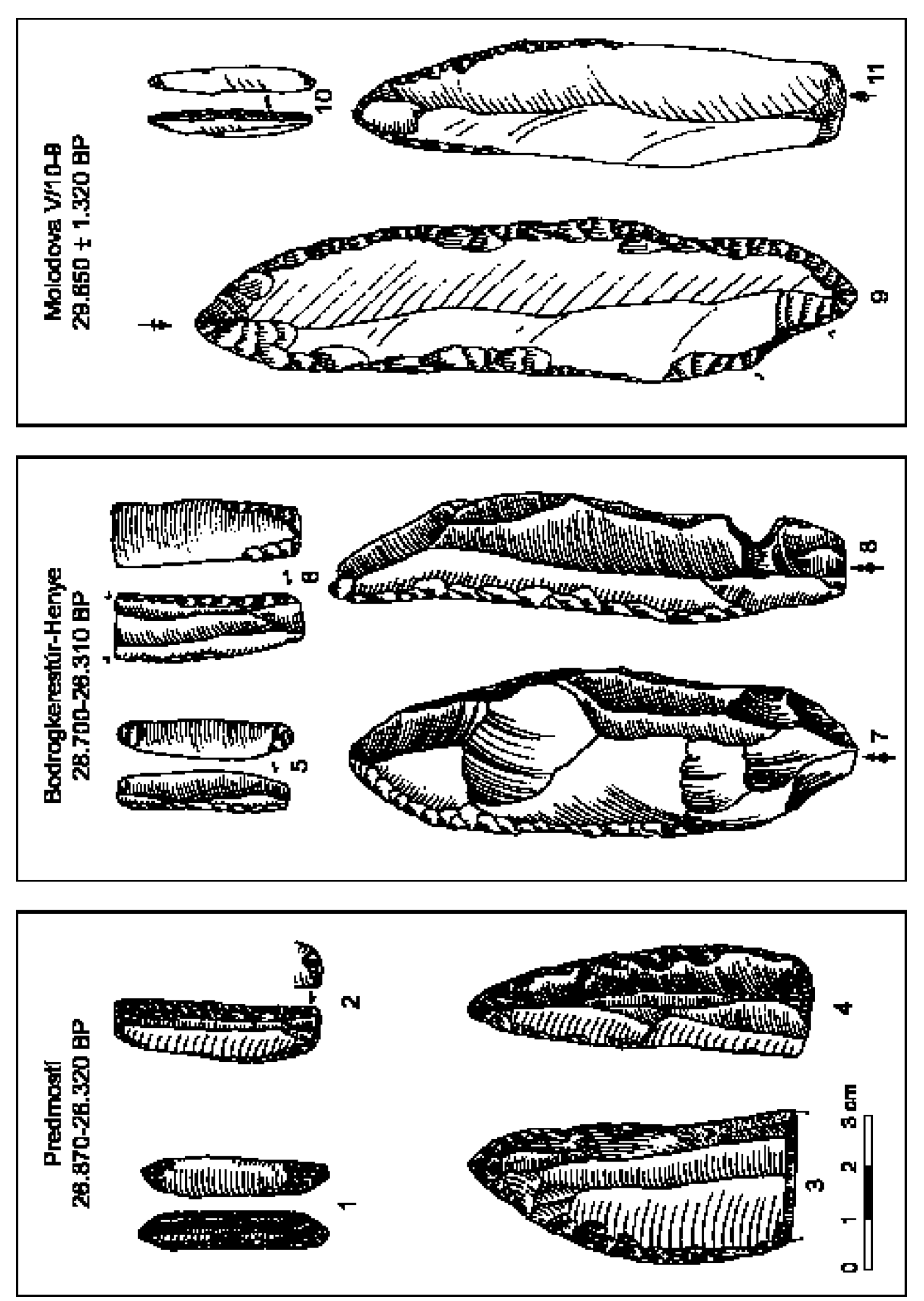

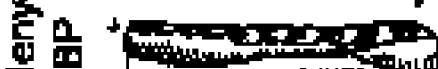

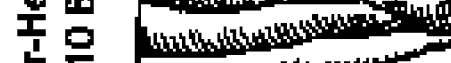

늘

象

严

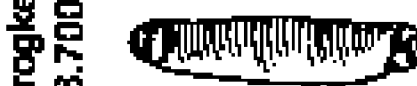

要

is
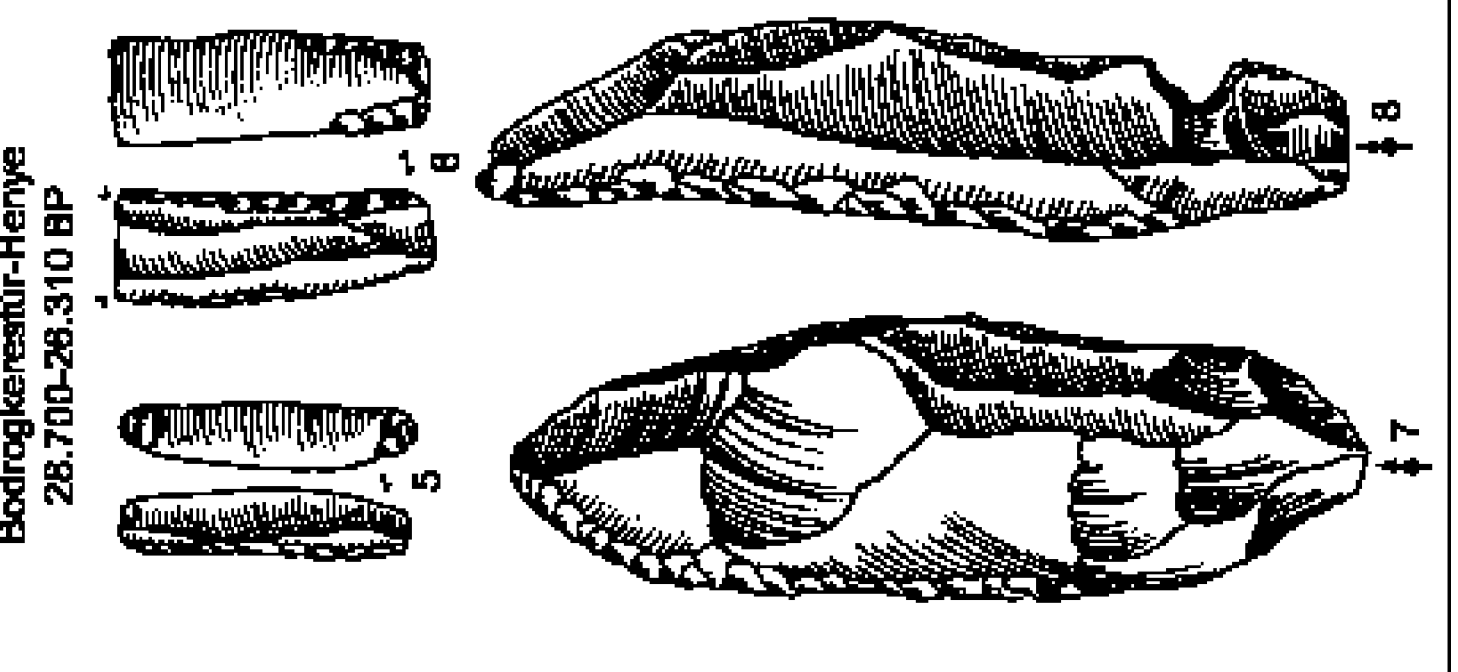

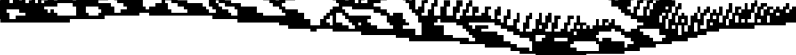




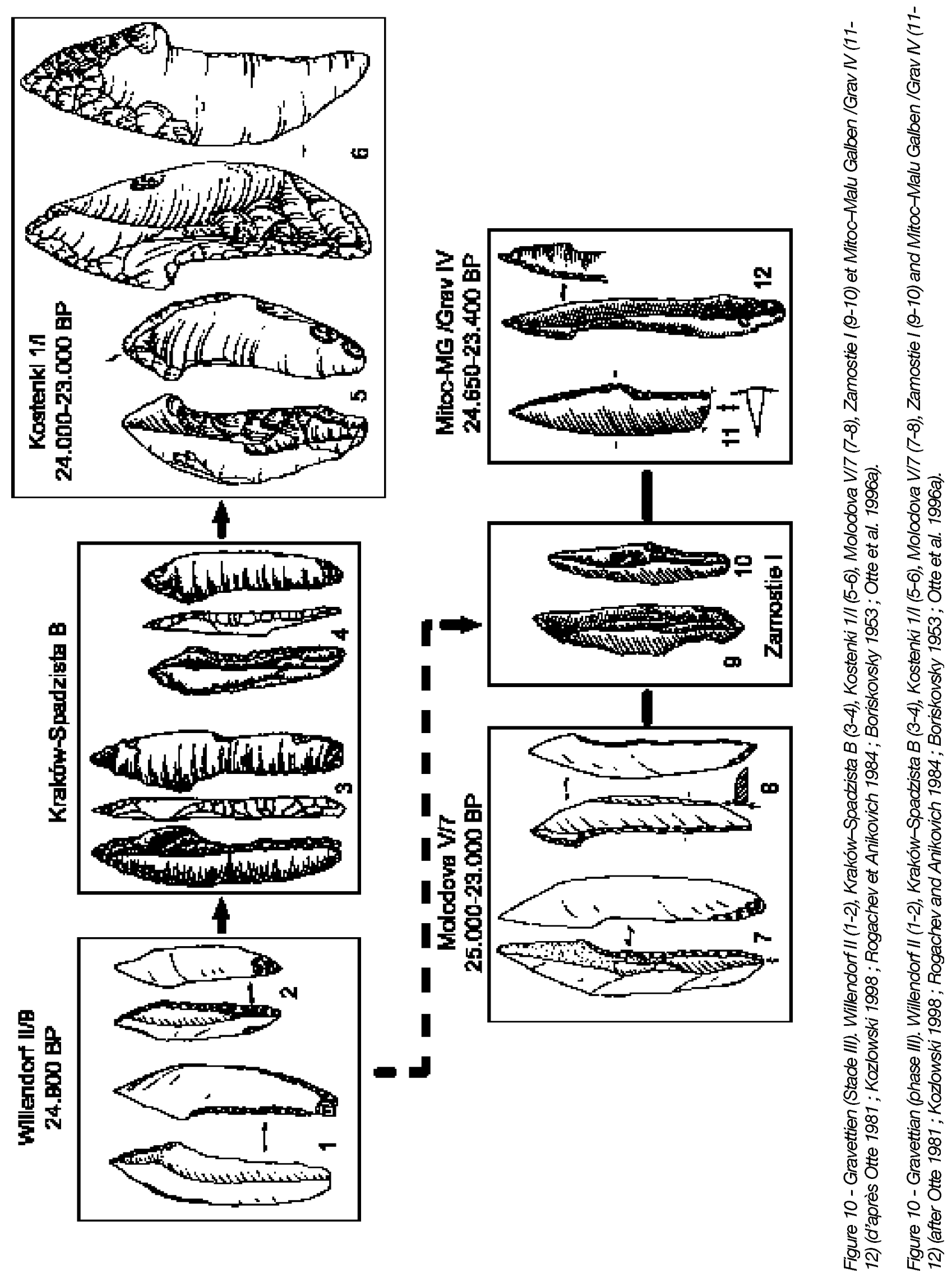




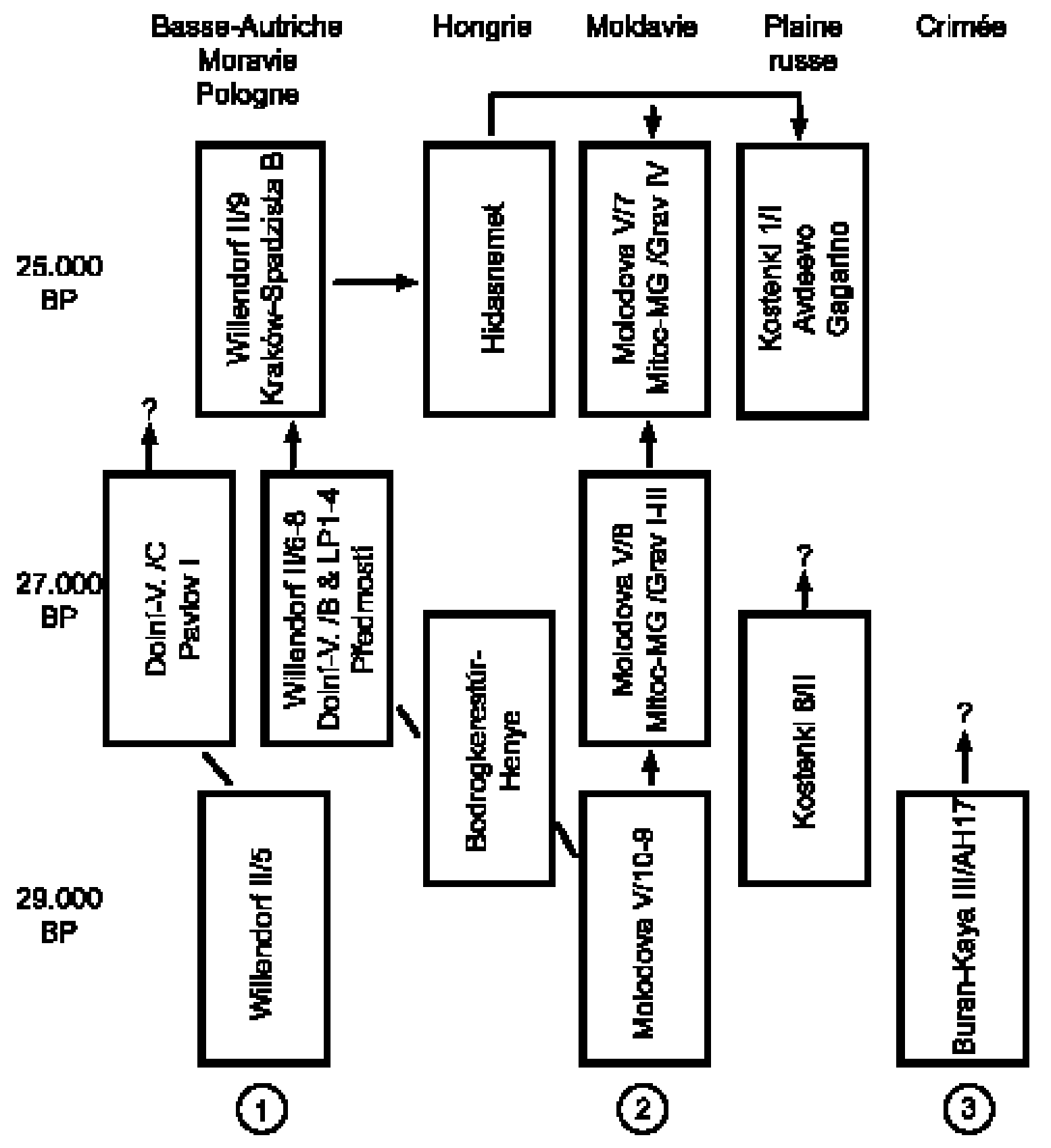

Figure 11 - Origines et évolution du Gravettien. Trois centres d'origine sont envisageables pour le Gravettien d'Europe centrale et orientale; leur influence sur les industries postérieures varie selon les régions considérées.

Figure 11 - Origin and development of the Gravettian. Three centres of origin are proposed for the Gravettian of Central and Eastem Europe; their influence on succeeding industries varies according to the regions considered. 


\section{6 - LE PLÉNIGLACIAIRE SUPÉRIEUR ENTRE 23000 ET 20000 BP ET LA SUITE DE LA TRADITION DES OUTILS À DOS}

Même si les conditions climatiques se détériorent durant cette période, il n'y a pas eu désaffection totale du territoire moldave. Quelques sites montrent de faibles traces d'occupation, manifestées par des objets épars (jamais diagnostiques) et des concentrations de cendres et de charbons de bois.

Quelques datations radiométriques proviennent de sites gravettiens et/ou épigravettiens (même si les artefacts lithiques associés à ces datations ne sont jamais diagnostiques). Par exemple, des charbons de bois associés à des artefacts en silex ont été retrouvés entre les niveaux culturels 7 et 6 de Molodova $V$, dans les unités stratigraphiques 13.1, 13.2 et 13.3 (Haesaerts et al. 2003) ; quatre dates ont été obtenues, comprises entre 21540 et 20610 BP. Une date similaire existe à Cotu-Miculinti (Paunescu 1989). Les données de Ciuntu, petit abri sous roche ayant livré peu de vestiges lithiques mais une grande quantité de restes fauniques datés entre 22100 et 18500 BP (Hedges et al. 1996 ; Borziac et al. 1997), permettent d'imaginer une fréquentation orientée vers la prédation, où l'abri était destiné aux travaux de boucherie (beaucoup d'ossements, très fracturés) plutôt qu'au travail du silex (peu de vestiges lithiques), avec des indices micro-morphologiques de stabilisation de la surface de la terrasse pendant une période assez longue, stabilisation peut-être responsable de l'accumulation de vestiges osseux d'âges différents.

À partir de 20000 BP, les occupations redeviennent nombreuses en Moldavie, ce dont témoignent d'abord le niveau 6 de Molodova V, daté de 20320 BP (Haesaerts et al. 2003), puis les niveaux 10,9 et suivants de Cosauti, vers 19400 BP (Otte et al. 1996b ; Haesaerts et al. 1998). Ce sont des ensembles épigravettiens dont les plus remarquables proviennent de Molodova V (Chernysh 1987), de Korman IV (Chernysh 1977) et de Cosauti (Borziac 1989, 1991, 1993a, 1993b).

La majorité des ensembles montre une remarquable homogénéité de leur spectre faunique (Chernysh 1959, 1987 ; Tatarinov 1977 ; Borziac 1993a). Le renne est l'espèce dominante absolue, autant par le nombre de restes que par le nombre d'individus. L'homogénéité des ensembles épigravettiens est perceptible dans les structures d'habitat, peu variées. Elles consistent en traces de petites huttes ou de cabanes de forme circulaire ou ovale, aménagées autour d'un foyer central, parfois pourvues d'un second foyer vers l'entrée. Alentour, s'organisent des aires d'activités spécialisées (débitage, boucherie, rejet). Ces structures légères évoquent un mode de vie résolument nomade.

Comme dans le Gravettien, le débitage épigravettien varie peu. Les supports des outils ne sont pas souvent retouchés. Le spectre typologique est limité à quelques classes d'outils, que l'on retrouve dans la majorité des industries (les burins sont les outils les plus nombreux, suivis par les lames retouchées et les grattoirs, puis les outils à dos, en nombre variable selon les ensembles). L'outillage osseux, déjà bien attesté dans le Gravettien, se développe et inclut des outils domestiques (aiguilles, houes, pics, pioches, marteaux) et des armatures (pointes de sagaie, pointes de projectile) (Borziac 1993a).
Il existe des différences entre les sites. Quelques-uns sont orientés vers l'acquisition de nourriture, d'autres vers le débitage ou vers le travail de l'os et du bois de renne. Les sites du Dniestr semblent par contre correspondre à des camps de base.

Les occupations épigravettiennes ont pris fin en Moldavie avec la sédimentation rapide et intense d'un lœss sableux entre 17200 et 16000 BP (bien attesté à Cosauti sur une puissance de $4 \mathrm{~m}$; Haesaerts et al. 1998).

L'Épigravettien présente en Moldavie toutes les caractéristiques d'une entité homogène à un moment du Paléolithique supérieur (20 000 - 17000 BP), au même titre que le Pavlovien auparavant en Moravie, et comme le Mézinien et l'Élissevichien pourront l'être ensuite dans les bassins du Dniepr et du Pripet (Otte et Noiret 2004).

Après la fin des occupations de l'Épigravettien ancien, le territoire moldave semble de nouveau abandonné. La phase aride immédiatement postérieure voit notamment l'installation de communautés dans les bassins du Dniepr et du Pripet, où se développement le Mézinien et l'Élissevichien. Des indices de réoccupation de la Moldavie apparaissent avec le Tardiglaciaire et une forme d'Épigravettien récent. Les industries attribuables à cette fourchette chronologique sont peu nombreuses. Nous y incluons les niveaux supérieurs (3 à 1) de Molodova $V$, en raison des dates radiométriques (Ivanova et Chernysh 1965). Les deux niveaux supérieurs de Korman IV les accompagnent probablement (Chernysh 1977 ; Ivanova 1977) ; ils n'ont pas été datés, mais leurs industries présentent des similitudes techno-typologiques avec celles de la partie supérieure de Molodova V. II est probable que de nombreux autres sites aient été contemporains de ces ensembles du Dniestr moyen. Ainsi, en est-il de plusieurs sites de Roumanie, non datés mais fréquemment rapportés au Tardiglaciaire ou comparés aux niveaux 3 à 1 de Molodova V (Paunescu 1984 ; Chirica 1989), tels Topile ou Movileni dans le sud de la Moldavie (en direction de Galati), ou de certains ensembles de la zone de Ceahlau (le niveau 6 de Bistricioarei-Lutarie, le niveau 5 de Ceahlau-Dîrtu, le niveau 2 de Ceahlau-Bofu Mic, les niveaux 3-5 de Ceahlau-Podis).

\section{7 - CONCLUSION}

La Moldavie n'a pas constitué une zone d'émergence majeure du Paléolithique supérieur ancien. Les niveaux 10 et 9 de Molodova $V$ semblent bien constituer l'une des premières manifestations gravettiennes en Europe, particulièrement en ce qui concerne le stade à grandes lames retouchées (Noiret, Engesser et Otte 1999). Ce stade semble antérieur à ses équivalents d'Europe centrale (fig. 9). Dans le cas du stade à pointes à cran, celles-ci témoignent peutêtre d'une influence extérieure, cependant limitée (fig. 10). Au final, l'évolution continue et locale du Gravettien en Moldavie semble bien assurée (fig. 11). Dans ce sens, le Molodovien se démarque des autres faciès gravettiens d'Europe centrale et orientale, le Pavlovien de Moravie, puis le Kostenkien de la Plaine russe : il a perduré plus longtemps, mais ses manifestations matérielles (esthétiques et rituelles y compris) ont été moins spectaculaires. II s'agit toutefois d'une variante tout aussi efficace. 
Après la période comprise entre 23000 et $20000 \mathrm{BP}$, correspondant à un important hiatus dans les occupations humaines, la majorité des ensembles culturels relève de l'Épigravettien, dont la filiation avec le Gravettien antérieur est sensible (réoccupation d'emplacements plus anciens à Ko man IV et Molodova $\mathrm{V}$, emploi des mêmes techniques d'aménagement des outils lithiques, développement de l'outillage osseux). Des différences apparaissent pourtant: les modes de subsistance sont plus variés (grands et petits mammifères, oiseaux, poissons et peut-être mollusques et végétaux) et surtout très spécialisés vers la prédation du renne, puis dans une moindre mesure du cheval.

\section{BIBLIOGRAPHIE}

ANIKOVICH M.V. 1992 - Early Upper Paleolithic industries of Eastern Europe. Journal of World Prehistory, 6(2), p. 205-245.

BORISKOVSKY P.I. 1953 - Le Paléolithique de I'Ukraine (Esquisse historique et archéologique). Moscou-Leningrad, Mater. Issl. Arkeol. S.S.S.R., 40 (en russe).

BORZIAC I.A. 1989 - Kosouti : a multi-level Late Palaeolithic site on the Middle Dniester, Moldavian SSR. Antiquity, 63, p. 791-792.

BORZIAC I.A. 1991 - Quelques données préalables sur l'habitat tardipaléolithique pluristratifié de Cosseoutsy sur le Dniestr moyen. In : V. Chirica et D. Monah (Ed.), Le Paléolithique et le Néolithique de la Roumanie en contexte européen. Iasi, Bibliotheca Archaeologica lassiensis IV, p. 56-71.

BORZIAC I.A. 1993a - Subsistence practices of Late Paleolithic groups along the Dnestr river and its tributaries. In : O. Soffer et N.D. Praslov (Ed.), From Kostenki to Clovis. Upper Paleolithic-Paleo-Indian Adaptations. New York-Londres, Plenum Press, p. 67-84.

BORZIAC I.A. 1993b - Les chasseurs de renne de Kosoioutsy, site Paléolithique tardif à plusieurs niveaux sur le Dniestr moyen (rapport préliminaire). L'Anthropologie, 97(2-3), p. 331-336.

BORZIAC I.A. 1994 - Paleoliticul si Mezoliticul în spatiul dintre Nistru si Prut. Thraco-Dacia, xv(1-2), p. 19-40.

BORZIAC I.A. 1998 - Le Gravettien de la région du Dniestr et ses liens avec le complexe Willendorf-Pavlov-Kostenki. In : H.A. Amirkhanov (Ed.), The Eastern Gravettian, Actes du Colloque de Moscou-Zaraysk (1-7 septembre 1997). Moscou, Russian Academy of Sciences (Institute of Archaeology), p. 135-141 (en russe).

BORZIAC I.A. et CHETRARU N.A. 1995 - Statiunea din Paleoliticul superior Ciutulesti I. A rheologia Moldovei, XVIII, p. 95-113.
BORZIAC I.A. et CHIRICA V. 1999 - Considérations concernant le Gravettien de l'espace compris entre le Dniestr et les Carpates. Préhistoire européenne, 14, p. 67-78.

BORZIAC I.A. et KULAKOVSKA L.V. 1998 - Le Gravettien de la zone du Dniestr. Synthèse. Archéologie (Kiev), 4, p. 55-63 (en ukrainien).

BORZIAC I.A., ALLSWORTH-JONES Ph., FRENCH C., MEDIANIK S.I., RINK W.J. et LEE H.K. 1997 - The Upper Palaeolithic site of Ciuntu on the Middle Pruth, Moldova : a mustidisciplinary study and reinterpretation. Proceedings of the Prehistoric Society, 63, p. 285-301.

CHERNYSH A.P. 1959 - Le Paléolithique tardif du bassin moyen du Dniestr. In: Le Paléolithique du bassin moyen du Dniestr. Moscou, Édition de l'Académie des Sciences de I'URSS (Travaux de la Commission pour l'Étude du Quatemaire XV), p. 5-214 (en russe).

CHERNYSH A.P. 1961 - Le site paléolithique de Molodova $V$. Kiev, 172 p. (en ukrainien).

CHERNYSH A.P. 1973 - Paleolithic and Mesolithic of the Dnestr Area (Maps and Catalogue of Sites). Moscou, Nauka, 127 p. (en russe).

CHERNYSH A.P. 1977 - Multilayer Paleolithic site Korman IV and its place in the Paleolithic. Dans: The Multilayer Paleolithic Site Korman IV on the Middle Dniestr. Moscou, Nauka, p. 7-77 (en russe).

CHERNYSH A.P. 1985 - Paléolithique supérieur. In : Archéologie de l'Ukraine. Vol. 1 : Archéologie préhistorique. Kiev, Naukova Dumka, p. 54-83 (en russe).

CHERNYSH A.P. 1987 - The standard multilayered site Molodova V. Archeology. In : I.K. Ivanova et S.M. Meitzin (Ed.), The Multilayered Paleolithic Site Molodova V. The Stone Age Men and Environment. Moscou, Nauka, p. 7-93 (en russe).

CHETRARU N.A. 1973 - Les sites de l'époque paléolithique et mésolithique. Cartes archéologiques de la République Moldave, Vol. 1. Chisinau, Stiinta, 177 p. (en russe).

CHIRICA V. 1982 - Amuleta-pandantiv de La Mitoc, jud. Botosani. Studii si Cercetari de Istorie veche si Arheologie, 33(2), p. 229-231.

CHIRICA V. 1989 - The Gravettian in the East of the Romanian Carpathians. Iasi, Bibliotheca Archaeologica lassiensis III, $239 \mathrm{p}$.

DAMBLON Fr., HAESAERTS P. et VAN DER PLICHT J. 1996 - New datings and considerations on the chronology of Upper Palaeolithic sites in the Great Eurasiatic Plain. Préhistoireeuropéenne, 9, p. 177-231.

DOBOSI V.T. 2000 - Archaeological investigations at Bodrogkeresztúr-Henye. Dans : V.T. Dobosi (éd.), 
Bodrogkeresztúr-Henye Upper Palaeolithic Site (NEHungary). Budapest, Magyar Nemzeti Múzeum, p. 5-106.

GIRIA E. et BRADLEY Br.A. 1998 - Blade technology at Kostenki 1/1, Avdeevo and Zaraysk. In : H.A. Amirkhanov (Ed.), The Eastern Gravettian, Actes du Colloque de Moscou-Zaraysk (1-7 septembre 1997). Moscou, Russian Academy of Sciences (Institute of Archaeology), p. 191-213.

GRIGOR'EV G.P. 1970 - Le Paléolithique supérieur. In: Kamennyi vek na territorii SSSR. Moscou, Nauka, p. 43-63 (en russe).

GUBIN S.V. 1977 - Buried Pleistocene soils of the site Korman IV. In : The Multilayer Paleolithic Site Korman IV on the Middle Dniestr. Moscou, Nauka, p. 98-104 (en russe).

HAESAERTS P., DAMBLON Fr., BACHNER M. et TRNKA G. 1996 - Revised stratigraphy and chronology of the Willendorf II sequence, Lower Austria. Archaeologia Austriaca, 80, p. 25-42.

HAESAERTS P., BORZIAC I.A., VAN DER PLICHT J. et DAMBLON Fr. 1998 - Climatic events and Upper Paleolithic chronology in the Dniestr basin : new $14 \mathrm{C}$ results from Cosautsi. Radiocarbon, 40(2), p. 649-657.

HAESAERTS P., BORZIAC I.A., CHIRICA V., DAMBLON Fr., KOULAKOVSKA L. et VAN DER PLICHT J. 2003 - The East Carpathian lœss record : a reference for the Middle and Late Pleniglacial stratigraphy in Central Europe. Quaternaire, 14(3), p. 163-188.

HAESAERTS P., BORZIAC I.A., CHIRICA V., DAMBLON Fr. et KOULAKOVSKA L. 2004 - Cadre stratigraphique et chronologique du Gravettien en Europe centrale. In: J. Svoboda et L. Sedlácková (éd.), The Gravettian along the Danube, Actes du Colloque de Mikulov (20-21 novembre 2002). Brno, Institute of Archaeology (The Dolní Vestonice Studies 11), p. 33-56.

HEDGES R.E.M., HOUSLEY R.A., PETTITT P.B., BRONK RAMSEY C. et VAN KLINKEN G.J. 1996 - Radiocarbon dates from the Oxford AMS System : Archaeometry datelist 21. Archaeometry, 38(1), p. 181-207.

HOFFECKER J.F. 1988 - Early Upper Paleolithic sites of the European USSR. In : J.F. Hoffecker et C.A. Wolf (éd.), The Early Upper Paleolithic. Evidence from Europe and the Near East. Oxford, BAR International Series 437, p. 237-272.

IVANOVA I.K. 1959 - Les conditions géologiques de découverte des stations paléolithiques du bassin moyen du Dniestr. In: Le Paléolithique du bassin moyen du Dniestr. Moscou, Édition de l'Académie des Sciences de I'URSS (Travaux de la Commission pour l'Étude du Quatemaire Xv), p. 216-278 (en russe).

IVANOVA I.K. 1971 - Stratigraphie des dépôts quaternaires et géologie du Paléolithique au Sud de la partie européenne de l'URSS. In: Études sur le Quatemaire dans le monde, Vol. 2. vIII Congrès de l'INQUA (Paris, 1969). Paris, CNRS, p. 661-664.

IVANOVA I.K. 1977 - Geology and paleogeography of the site Korman IV on the general background of the geological history of the Paleolithic Middle Dniestr. In : The Multilayer Paleolithic Site Korman IV on the Middle Dniestr. Moscou, Nauka, p. 126-181 (en russe).

IVANOVA I.K. 1987 - Paleogeography and paleoecology of the environment of stone age men inhabitance in the Middle Dniestr. Site of Molodova V. In : I.K. Ivanova et S.M. Meitzin (éd.), The Multilayered Paleolithic Site Molodova V. The Stone Age Men and Environment. Moscou, Nauka, p. 94-123 (en russe).

IVANOVA I.K. et CHERNYSH A.P. 1965 - The Paleolithic site of Molodova $V$ on the Middle Dnestr (USSR). Quatemaria, vII, p. 197-217.

KOZLOWSKI J.K. 1986 - The Gravettian in Central and Eastem Europe. In : Fr. Wendorf et A.E. Close (Ed.), Advances in World Archaeology 5. Orlando, Academic Press, p. 133-200.

KOZLOWSKI J.K. 1996 - L'origine du Gravettien dans le Sud-Est européen. In : A. Montet-White, A. Palma di Cesnola et K. Valoch (Ed.), The Upper Palaeolithic. Colloquium XII : The Origin of the Gravettian, Actes du XIII Congrès international de I'UISPP (Forlì, 8-14 septembre 1996), série Colloquia (Vol. 6). Forlì, ABACO, p. 191-202.

KOZLOWSKI J.K. 1998 - La géochronologie de l'horizon à pointes à cran en Europe centrale. In : H.A. Amirkhanov (éd.), The Eastern Gravettian, Actes du Colloque de Moscou-Zaraysk (1-7 septembre 1997). Moscou, Russian Academy of Sciences (Institute of Archaeology), p. 81-89.

KOZLOWSKI J.K. 2000 - Châtelperronien, Uluzzien et quoi plus à l'Est ? Anthropologie, XXXVIII(3), p. 249-259.

LÓPEZ BAYÓN I. et GAUTIER A., 2007 - Mitoc-Malu Galben, analyse archéo-zoologique des ateliers de taille. In : M. Otte et V. Chirica (Dir.), Mitoc-Malu Galben. Liège, Université de Liège (ERAUL 72), p. 145-166

MOGOSANU FI. 1983 - Paléolithique et Épipaléolithique. In : V. Dumitrescu, A. Bolomey et Fl. Mogosanu, Esquisse d'une préhistoire de la Roumanie jusqu'à la fin de l'âge du Bronze. Bucarest, Editura stiintifica si Enciclopedica, p. 29-55.

MOGOSANU FI. 1986 - Despre stratigrafia si periodizarea Gravetianului din Moldova. Studii si Cercetari de Istorie veche si Arheologie,37(2), p. 159-162.

MOTUZ V.M. 1977 - On the fauna of continental mollusks of the region of the site Korman IV. In : The Multilayer Paleolithic Site Korman IV on the Middle Dniestr. Moscou, Nauka, p. 119-125 (en russe). 
MURARU A. 1990 - Le gisement de silex de la vallée du $P$ rut, source de matière première pour l'outillage lithique dans la Préhistoire. Étude monographique préliminaire. In : M.-R. Séronie-Vivien et M. Lenoir (dir.), Le silex, de sa genèse à l'outil, Actes du $5^{\mathrm{e}}$ Colloque international sur le silex (Bordeaux, 17 sept.-2 oct. 1987). Paris, CNRS (Cahiers du Quatemaire 17), t. I, p. 149-159.

NICOLAESCU-PLOPSOR C.S., PAUNESCU Al. et MOGOSANU FI. 1966 - Le Paléolithique de Ceahlau. Dacia, x, p. 2-116.

NOIRET P. 2003-2004 - Le Paléolithique supérieur de la Moldavie. Essai de synthèse d'une évolution multi-culture / Ie. Thèse de Doctorat (non publiée), Université de Liège, 3 vol., $645 \mathrm{p}$.

NOIRET P., ENGESSER K. et OTTE M., 1999 - Proposition de révision des stades techno-typologiques du Gravettien oriental. In : J. Evin, Chr. Oberlin, J.-P. Daugas et J.-Fr. Salles (éd.), $14 \mathrm{C}$ et Archéologie, Actes du $3^{\mathrm{e}}$ Congrès intemational de Lyon (6-10 avril 1998). Paris-Rennes, Mémoires de la Société préhistorique française (t. XXVI) - Supplément 1999 à la Revue d'Archéométrie, p. 151-155.

OTTE M. 1981 - Le Gravettien en Europe centrale. Bruges, De Tempel (Dissertationes Archaeologicae Gandenses XX), 2 vol., 505 p.

OTTE M. 1990 - Révision de la séquence du Paléolithique supérieur de Willendorf (Autriche). Bulletin de l'Institut royal des Sciences naturelles de Belgique. Sciences de la Terre, 60 , p. $219-228$.

OTTE M. 1998 - Aspects du Gravettien hongrois. Acta Archaeologica Academiae Scientianum Hungaricae, 50, p. 1-27.

OTTE M. et NOIRET P. 2004 - Évolution du Gravettien au Moyen Danube. In : J. Svoboda et L. Sedlácková (Ed.), The Gravettian along the Danube, Actes du Colloque de Mikulov (20-21 novembre 2002). Brno, Institute of Archaeology (The Dolní Vestonice Studies 11), p. 8-32.

OTTE M., NOIRET P., CHIRICA V. et BORZIAC I.A., 1996a. Rythme évolutif du Gravettien oriental. In : A. Montet-White, A. Palma di Cesnola et K. Valoch (Ed.), The Upper Palaeolithic. Colloquium XII : The Origin of the Gravettian, Actes du XIIle Congrès international de I'UISPP (Forlì, 8-14 septembre 1996), série Colloquia (Vol. 6). Forlì, ABACO, p. 213-226.
OTTE M., LÓPEZ BAYÓN I., NOIRET P., BORZIAC I.A. et CHIRICA V., 1996b. Recherches sur le Paléolithique supérieur de la Moldavie. Bulletin de la Société royale belge Anthropologie et Préhistoire, 107, p. 45-80.

OTTE M., NOIRET P., CHIRICA V. et BORZIAC I.A. 2007 Étude de l'industrie lithique. In : M. Otte et V. Chirica (dir.), Mitoc-Malu Galben. Liège, Université de Liège (ERAUL 72), p. 85-135.

PAUNESCU Al. 1970 - Evolutia uneltelor si armelor de piatra cioplita descoperite pe teritiriul României. Bucarest, Editura Academiei Republicii Socialiste România, 359 p., 60 fig., nbr. tabl. (Biblioteca de Arheologie XV).

PAUNESCU Al. 1984 - Cronologia Paleoliticului si Mezoliticului din România în contextul Paleoliticului central-est si sud European. Studii si Cercetari de Istorie veche si Ameologie, 35(3), p. 235-265.

PAUNESCU Al. 1989 - Le Paléolithique et le Mésolithique de Roumanie (Un bref aperçu). L'Anthropologie, 93(1), p. 123-158.

PETTITT P.B. 1998 - Middle and Early Upper Palaeolithic Crimea : the radiocarbon chronology. In : M. Otte (dir.), Préhistoired'Anatolie. Genèse de deux mondes, Actes du Colloque international de Liège (28 avril-3 mai 1997). Liège, Université de Liège (ERAUL 85), vol. I, p. 329-338.

ROGACHEV A.N. et ANIKOVICH M.V. 1984 - Le Paléolithique supérieur de la Plaine russe et de la Crimée. In : P.I. Boriskovsky (éd.), Paleolit SSSR. Moscou, Nauka, p. 162-271 (en russe)

TATARINOV K.A. 1977 - Fauna of vertebrates of the site Korman IV. In : The Multilayer Paleolithic Site Korman IV on the Middle Dniestr. Moscou, Nauka, p. 112-118 (en russe).

VALOCH K. 1984 - Transition du Paléolithique moyen au Paléolithique supérieur dans l'Europe centrale et orientale. In : Scripta Praehistorica. Oblata Francisco Jorda. Salamanca, p. 439-467.

VELICHKO A.A., BOGUCKI A.B., MOROZOVA T.D., UDARTSEV V.P., KHALCHEVA T.A. et TSASKIN A.I., 1984. Periglacial landscapes of the East European Plain. In : A.A. Velichko (Ed.), Late Quatemary Environments of the Soviet Union. Minneapolis, University of Minnesota Press, p. 95-118.

YANEVICH A.A., STEPANCHUK V.N. et COHEN V.Y., 1996. Buran-Kaya III and Skalistiy rockshelter : two new dated Late Pleistocene sites in the Crimea. Préhistoireeuropéenne, 9, p. 315-324. 
